\title{
Article \\ Forecasting Air Temperature on Edge Devices with Embedded AI ${ }^{+}$
}

\author{
Gaia Codeluppi (D), Luca Davoli (D) and Gianluigi Ferrari *(D)
}

Citation: Codeluppi, G.; Davoli, L.; Ferrari, G. Forecasting Air Temperature on Edge Devices with Embedded AI. Sensors 2021, 21, 3973. https://doi.org/10.3390/s21123973

Academic Editors: Davide Brunelli and Annachiara Berardinelli

Received: 12 April 2021

Accepted: 7 June 2021

Published: 9 June 2021

Publisher's Note: MDPI stays neutral with regard to jurisdictional claims in published maps and institutional affiliations.

Copyright: (c) 2021 by the authors. Licensee MDPI, Basel, Switzerland. This article is an open access article distributed under the terms and conditions of the Creative Commons Attribution (CC BY) license (https:/ / creativecommons.org/licenses/by/ $4.0 /)$.
Internet of Things (IoT) Lab, Department of Engineering and Architecture, University of Parma, Parco Area delle Scienze, 181/A, 43124 Parma, Italy; gaia.codeluppi@unipr.it (G.C.); luca.davoli@unipr.it (L.D.)

* Correspondence: gianluigi.ferrari@unipr.it; Tel.: +39-0521-906513

+ This paper is an extended version of our paper published in AI at the Edge: A Smart Gateway for Greenhouse Air Temperature Forecasting. In Proceedings of the 2020 IEEE International Workshop on Metrology for Agriculture and Forestry (MetroAgriFor), 38122 Trento, Italy, 4-6 November 2020.

\begin{abstract}
With the advent of the Smart Agriculture, the joint utilization of Internet of Things (IoT) and Machine Learning (ML) holds the promise to significantly improve agricultural production and sustainability. In this paper, the design of a Neural Network (NN)-based prediction model of a greenhouse's internal air temperature, to be deployed and run on an edge device with constrained capabilities, is investigated. The model relies on a time series-oriented approach, taking as input variables the past and present values of the air temperature to forecast the future ones. In detail, we evaluate three different NN architecture types—namely, Long Short-Term Memory (LSTM) networks, Recurrent NNs (RNNs) and Artificial NNs (ANNs) - with various values of the sliding window associated with input data. Experimental results show that the three best-performing models have a Root Mean Squared Error (RMSE) value in the range $0.289 \div 0.402{ }^{\circ} \mathrm{C}$, a Mean Absolute Percentage Error (MAPE) in the range of $0.87 \div 1.04 \%$, and a coefficient of determination $\left(\mathrm{R}^{2}\right)$ not smaller than 0.997. The overall best performing model, based on an ANN, has a good prediction performance together with low computational and architectural complexities (evaluated on the basis of the NetScore metric), making its deployment on an edge device feasible.
\end{abstract}

Keywords: internet of things; smart farming; EdgeAI; neural networks; greenhouse management; wireless sensor network; WSN; RNN; LSTM; ANN

\section{Introduction}

The introduction of Information and Communication Technologies (ICTs) in the agricultural sector, aiming to improve productivity and sustainability, is currently a wellestablished practice. Indeed, the integration of heterogeneous technologies such as, just to name a few, Internet of Things (IoT) and Machine Learning (ML), allows us to simplify and enhance the management of the so-called Smart Farms [1]. Due to this trend of technological transformation, usually denoted as Smart Agriculture (SA) or Smart Farming (SF), in recent years, greenhouses' productive processes have been optimized, for example in terms of increasing automatization.

To this end, greenhouses play a crucial role in worldwide agricultural productions. Indeed, through the creation of optimal growing conditions for indoor cultivation [2], vegetables, fruits, herbs and other kinds of edible products can be farmed anytime and everywhere, regardless of their seasonality or the (eventually adverse) weather conditions of their growing area. Therefore, the adoption of greenhouses offers various advantages, such as the extension of the cultivation periods of seasonal crops, the local production of non-endemic food, and reduced resource consumption, in terms of water, land and pesticides [3].

One of the most relevant (and challenging) aspects of greenhouse management is the development and maintenance of suitable growing habitats for the inside plants. This 
internal habitat, also referred to as "inner climate" or "micro-climate," corresponds to the complex set of environmental variables internal to the greenhouse (e.g., soil moisture, air humidity and temperature, and solar radiation) affecting the inner products' growth and depends on several interrelated elements [4]. In detail, there are (i) internal factors, such as, for example, the greenhouse' size and the cooling, warming or ventilation systems installed internally (thus referring to the greenhouse's actuators), and (ii) external factors (or "variables"), such as weather meteorological conditions (e.g., wind speed, solar radiation, temperature, and humidity) [5].

Nowadays, monitoring and controlling a greenhouse's micro-climate has been simplified and automatized thanks to the integration of heterogeneous technologies, which can be grouped into three categories.

- $\quad$ First, (sensor) data related to relevant environmental variables internal to the greenhouse, which have to be maintained within suitable ranges (e.g., air humidity and temperature), are collected through devices equipped with sensors (denoted as IoT sensing nodes, or sensor nodes, SNs), generally organized as Wireless Sensor Networks (WSNs). Moreover, internal greenhouse data gathered by SNs are usually sent to less constrained nodes, denoted as gateways (GWs) and connected to the Internet. GWs forward SNs' data to processing and storing infrastructures located in the Cloud [6]. Then, data can be retrieved and visualized (through appropriate User Interfaces, UIs), as well as kept as input data for further processing. Hence, monitoring of relevant variables inside the greenhouse is relevant for both end-users (farmers) and for researchers [7-11].

- Secondly, additional control devices (i.e., actuator nodes), installed inside the greenhouse in order to regulate its internal climate $[12,13]$, can be integrated within the aforementioned collection system. As an example, if a dangerous air humidity index is detected by SNs, a ventilation system would automatically be activated in order to lower the air humidity.

- Thirdly, complex models and/or forecasting algorithms are developed with the goal of predicting the future values of the monitored environmental variables, for example allowing us to preemptively schedule some operations (e.g., the activation of a warming system) to avoid these internal variables reaching undesired conditions (i.e., too low temperatures). To this end, the greenhouse's internal variables have been satisfactorily forecast through Deep Learning (DL) algorithms, e.g., based on Neural Networks (NNs) [14-16], and selecting data collected from different sources as input (namely, internal and external variables of a greenhouse, possibly measured by SNs).

For the sake of completeness, NN-based prediction algorithms can also be employed to infer missing sensor data, such as those not properly gathered inside the greenhouse due to a temporary lack of network connectivity, as well as to maintenance operations.

Since the adoption of NN-based algorithms generally provides significant benefits, they are more and more often integrated with IoT technologies in the context of SA. Moreover, although in recent years, these algorithms are mainly intended to be executed in the Cloud, a new promising IoT trend, denoted as EdgeAI and related to the transfer of intelligence (e.g., AI algorithms) from the Cloud down to the Edge (e.g., on IoT edge devices), is emerging [17]. Indeed, the execution of AI algorithms on IoT edge devices, which are located near the data sources (namely, SNs), provides relevant advantages, such as the reduction of the amount of data to be forwarded by IoT edge devices to the Cloud, thus reducing the network load, lowering the response latency, and, finally, supporting scalability. As a drawback, algorithms executed on-the-Edge have to be designed taking into account the limitations imposed by constrained IoT devices, with memory footprint, computational, and energy resources significantly lower than those offered by Cloud platforms. Thus, while modeling EdgeAI algorithms, there is the need to look for a balanced trade-off between reaching the best prediction performance and implementing a prediction model computationally "lightweight enough" to be executed by a constrained device (e.g., the required memory occupation has to be compatible with the one available 
on the device). In order to quantify the trade-off involved in achieving the two contrasting goals, in [18] the authors propose the NetScore metric, which is intended as a quantitative relative assessment of the prediction performance with computational and architectural complexities of a Deep NN (DNN).

In order to improve the management of greenhouses and to control their internal variables, in [19] — being the paper "AI at the Edge: a Smart Gateway for Greenhouse Air Temperature Forecasting" presented in the IEEE International Workshop on Metrology for Agriculture and Forestry (MetroAgriFor) — the authors have presented a novel approach based on the joint adoption of NNs, Edge Computing, and IoT technologies. In detail, the authors have "enriched" an IoT device, denoted as Smart GW and acting as a GW for a WSN installed in a greenhouse, in order to: (i) monitor its inner air temperature through an "edge intelligent" approach; (ii) locally forecast future inner air temperature's values; and (iii) regulate, according to the obtained results, the greenhouse air temperature (through internal actuators). From an algorithmic point of view, the authors have designed a lightweight device-friendly forecasting model, based on a fully-connected Artificial NN (ANN), able to predict the air temperature inside a greenhouse, also on the basis of the outside weather conditions.

In order to extend the solution proposed in [19], in the current paper, the authors evaluate an alternative approach to build an air temperature forecasting model to be executed on the Smart GW presented in [19] (with similar purposes). In particular, rather than relying on meteorological weather conditions (collected outside the greenhouse), the aim is now on the exploitation of air temperature values collected inside the greenhouse to forecast the air temperature evolution. Moreover, the authors evaluate the performance (in terms of prediction precision and lightweightness) of the model developed following a novel approach, denoted as time series-oriented, with respect to the model proposed in [19]. Then, the authors investigate how the model's parameters-namely, the number of input variables (i.e., air temperature values), their sampling interval, and the NN architectureinfluence the model's prediction performance. In detail, the following three types of NN architectures (which, according to the literature, outperform all other types of NNs for time series-based forecasting) have been evaluated: ANN, Recurrent NNs (RNNs), and Long Short-Term Memory (LSTM) networks. Finally, a comparison between the developed models and relevant approaches proposed in the literature for the same task (namely, air temperature forecasting inside greenhouses) is performed, in terms of prediction performance considering three metrics widely adopted in regression problems: (i) Root Mean Squared Error (RMSE); (ii) Mean Absolute Percentage Error (MAPE); and (iii) coefficient of determination $\left(\mathrm{R}^{2}\right)$. Unlike the typical approach followed in the literature, the authors also focus on the performance analysis in relative terms with respect to computational and architectural complexities. More precisely, this goal is achieved by relying on the use of the NetScore metric (in order to evaluate the complexity of the NN-based models to be run on constrained devices) - to the best of the authors' knowledge, this has never been considered before. This is fundamental in order to identify the proper model for IoT edge devices, but seldom discussed in the literature in the context of greenhouses' internal variables forecasting.

The rest of the paper is organized as follows. Section 2 gives an overview of NNs and an evaluation metrics adopted in this paper. In Section 3, a review on literature works is presented, while in Section 4 the adopted methodology is described. In Section 5, the experimental results obtained with the proposed models are outlined and discussed. Finally, in Section 6 some conclusions are drawn. To conclude, the methodological steps followed in this manuscript to perform target analysis and select the proper EdgeAI algorithm (to be deployed on the Smart GW) are summarized in Figure 1, with reference to the paper's internal structure. 


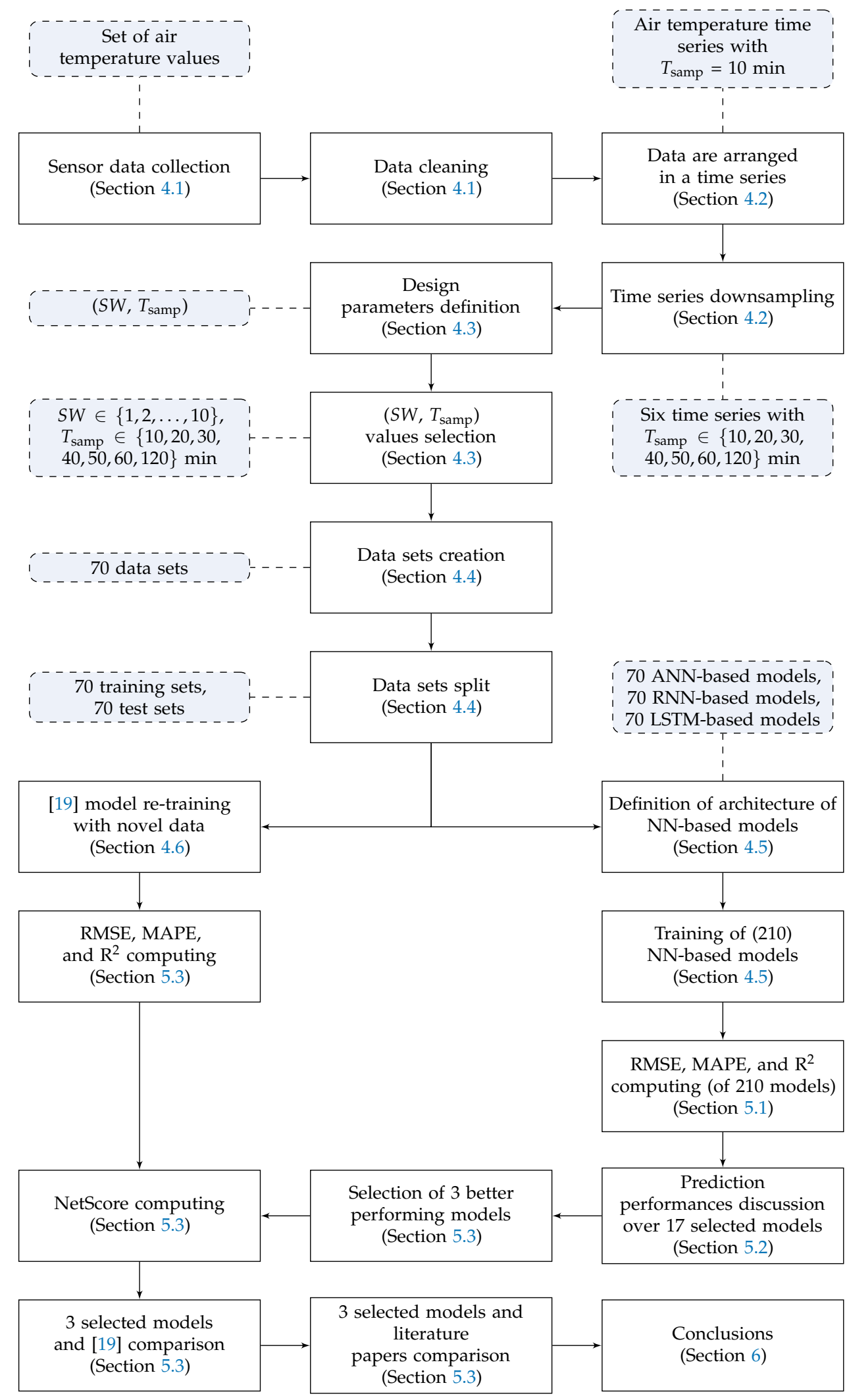

Figure 1. Methodological steps (white rectangles) and corresponding outcomes (violet rectangles with rounded corners) of this paper, with reference to its internal structure. 


\section{Background}

\subsection{Overview on Neural Networks}

In general terms, NNs can be seen as the "backbone" required to build ML-oriented algorithms that start from a set of data (or data set) and fit them into a parametric model [20-22], in order to solve different tasks, such as prediction [23]. More precisely, the parameters of these models are learnt from a group of samples-composing a training set-in a (preliminary) training phase, while their effectiveness is assessed on a samples test set in a (consecutive) test phase, adopting one or more evaluation metrics (as will be discussed in Section 2.2).

Due to their ability to (i) discover complex relations among data, (ii) be robust against data uncertainty, and (iii) predict output variables' values almost in real-time, NNs are appealing in many application areas, including greenhouse's inner variables' forecasting. As a drawback, in order to obtain an accurate forecasting model, NNs normally require a sufficiently large number of available data (sometimes not easy to be gathered) representative of the model to build.

Despite several types of NN-based architecture proposed in the literature (differing in terms of building elements, interconnections, and learning algorithms), a simple type is given by the Multi-Layer Perceptron (MLP) NN [24], in the following simply denoted as ANN. In detail, the model is built on top of multiple and interconnected processing units, denoted as neurons (or simply network nodes), organized in layers. Then, its internal organization allows input data to be processed from the first stages of the network to the final ones, thus allowing information to flow across the network, producing output variables. Moreover, models in which the data stream inside the network is linearly processed from the first layer (denoted as input layer) through one or more internal layers (denoted as hidden layers), till the final layer (denoted as output layer), are labeled as feed-forward. Furthermore, in the case that each node of a network layer is connected with every node of the previous layer, the network is denoted as fully-connected. Although an exhaustive discussion of the training process of a NN (from a mathematical point of view) is out of the scope of this paper, in the following the authors provide some high-level considerations, especially regarding the structure of a fully-connected feed-forward ANN.

Each neuron of the network (except for the input layer) receives information from all neurons in the previous layer (or from a subset, if the ANN is not fully-connected). In Figure 2, a simplified mathematical model describing the $j$-th neuron of an intermediate layer is shown. The messages arriving from the $\ell$ connected neurons from the previous layer, denoted as $\left\{x_{i}\right\}_{i=1}^{\ell}$, are combined within the $j$-th neuron through proper parametric functions, the most popular one being based on a weighted sum, with weights $\left\{w_{j i}\right\}_{i=1}^{\ell}$, of the input messages. In detail, each $j$-th neuron's input $x_{i}$ is multiplied by a weight $w_{j i}$ whose value is learnt during the ANN training phase (thus not a-priori fixed) and, then, added to the other weighed inputs messages. Then, a bias term $b_{j}$ is added to the sum. Finally, before being forwarded to the next ANN's layer, the output of a neuron is processed by a non-linear activation function, denoted as $f(\cdot)$ : a relevant example is the Rectified Linear Unit (ReLU) function $f(x)=\max (0, x)$ [21]. The overall output of the $j$-th neuron, denoted as $y_{j}$, can then be expressed as follows:

$$
y_{j}=f\left(\sum_{i=1}^{\ell} w_{j i} x_{i}+b_{j}\right) .
$$




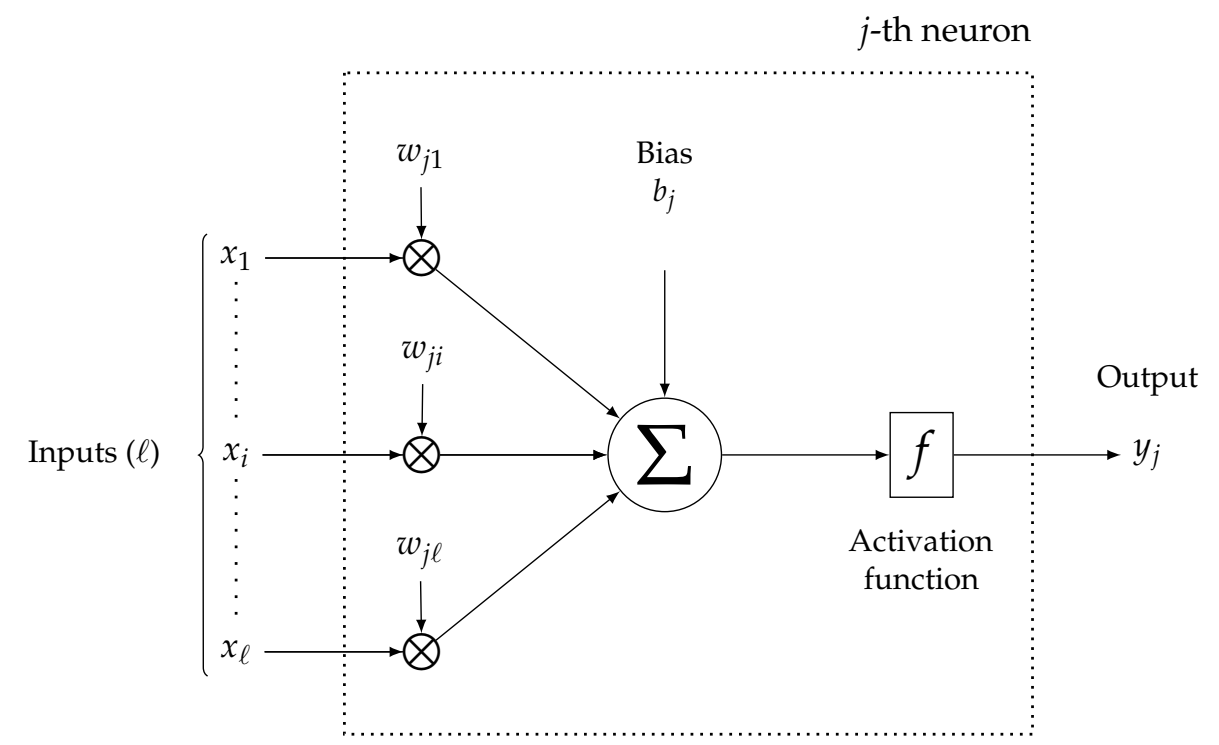

Figure 2. Simplified mathematical model of the processing of the $j$-th neuron inside a MLP ANN.

The training process of a NN is usually pursued through several rounds in which, according to a cost function quantifying the similarity between the NN's outputs and its desired values, the weights (in other words, the model's parameters) are updated to improve the performance or, more precisely, to reduce a cost function. To this end, there exist several algorithms implementing the learning process, among which one of the most popular ones is Back Propagation (BP) with gradient descent [23].

In the context of variable prediction in a greenhouse, beside ANNs, RNN and LSTM networks have been successfully applied to forecast air temperature (as detailed in Section 3). Indeed, due to their ability in discovering a connection between temporallyclose data, RNNs and LSTM networks are in general valid alternatives for solving time series-based forecasting problems. From a high-level perspective, this is mainly due to the fact that, for each stage in the training phase, a simple RNN can remember its internal state (namely, the output values of each of its neurons, as outlined in Figure 3) and exploit this state to compute the next state in the following round of training. Therefore, this internal organization allows us to analyze data in a recurring fashion, performing loops on data and resulting in a sort of short-term memory.

Recurrent Neural Network

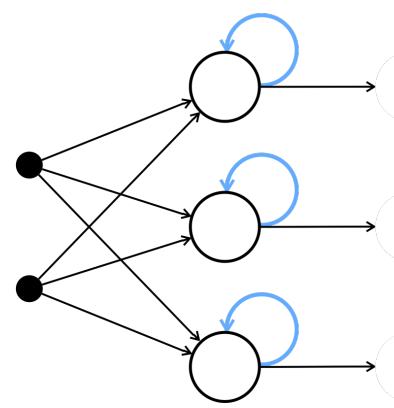

Feed-forward Neural Network

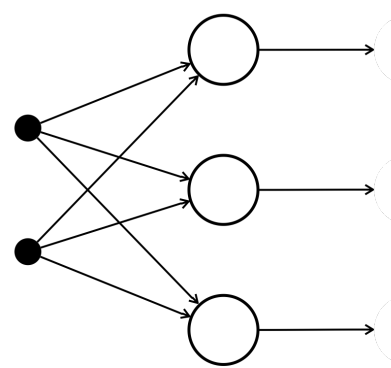

Figure 3. Comparison between the internal organization of a RNN, owing a sort of internal memory, and an ANN.

In order to overcome some limitations of RNNs, such as a vanishing gradient with long time series, which makes it difficult to learn long-term dependencies from data using RNN [25], valid alternatives are LSTM networks [26] (or simply LSTMs). From a conceptual point of view, LSTMs are built around a gated cell in which weights, learned during the training phase, define which information is more relevant to remember, thus 
allowing us to decide which value needs to be stored inside the (memory of the) cell and which one needs to be discarded. This allows us to select which information provided by the previous network's training round should be kept and which should not, better highlighting long-term relations among data.

\subsection{Evaluation Metrics}

The quality of a forecasting model, evaluated in terms of prediction performance in a comparative way with respect to multiple models devoted to the same task, is usually quantified selecting one or more proper metrics. In regression problems, such as the forecasting of future air temperature values inside a greenhouse, widely considered evaluation metrics are RMSE, MAPE, and $\mathrm{R}^{2}$ [21], defined as follows:

$$
\begin{aligned}
\text { RMSE } & =\sqrt{\frac{\sum_{j=1}^{n}\left(d_{j}-p_{j}\right)^{2}}{n}} \\
\text { MAPE } & =\frac{1}{n} \sum_{j=1}^{n}\left|\frac{d_{j}-p_{j}}{d_{j}}\right| \times 100 \\
\mathrm{R}^{2} & =\left[\frac{\sum_{j=1}^{n}\left(d_{j}-\bar{d}\right)\left(p_{j}-\bar{p}\right)}{\sqrt{\sum_{j=1}^{n}\left(d_{j}-\bar{d}\right) \sum_{j=1}^{n}\left(p_{j}-\bar{p}\right)}}\right]^{2}
\end{aligned}
$$

where $n$ is the number of predicted (and corresponding actual) samples; $\left\{d_{j}\right\}$ and $\left\{p_{j}\right\}$ are the actual and forecast values of the $j$-th sample of the phenomenon under observation (e.g., the temperature), respectively; and $\bar{d} \triangleq\left(\sum_{j=1}^{n} d_{j}\right) / n$ and $\bar{p} \triangleq\left(\sum_{j=1}^{n} p_{j}\right) / n$ are the average values of the actual and forecast samples, computed as arithmetic averages over all the $n$ actual and forecast samples, respectively.

The RMSE, defined according to Equation (2), represents the standard deviation of the difference between the actual values (to be predicted) and the values predicted by the model (or residuals). The closer to zero the RMSE is, the better the algorithm performs.

The MAPE, defined as in Equation (3), is a percentage quantifying a relative distance between predicted and actual values. As for the RMSE, the closer to zero the MAPE distance, the better the performance.

Finally, $R^{2}$, defined according to Equation (4), is a normalized (between 0 and 1) statistical metric quantifying the adaptability of a regression model. The closer to one $\mathrm{R}^{2}$ is, the more accurate the prediction model is.

A recently proposed metric, relevant for the evaluation of a $N N \mathcal{N}$ to be run on an edge device, is the NetScore metric [18], denoted as $\Omega$ and expressed as follows:

$$
\Omega(\mathcal{N})=20 \log \left(\frac{a(\mathcal{N})^{\alpha}}{p(\mathcal{N})^{\beta} \times m(\mathcal{N})^{\gamma}}\right)
$$

where $a(\mathcal{N})$ is the accuracy of the model (i.e., the correct prediction rate in a classification task); $p(\mathcal{N})$ is the number of parameters of a $\mathrm{NN}$, also denoted as architectural complexity; $m(\mathcal{N})$ is the number of Multiply-ACcumulate (MAC) operations performed during NN inference, also denoted as computational complexity; and $\alpha, \beta, \gamma$ are coefficients which allow to control the relative weights (for the purpose of NetScore evaluation) of accuracy, architectural complexity, and computational complexity of the model $\mathcal{N}$, respectively.

As can be seen from Equation (5), the NetScore metric assigns to an NN a score that is logarithmically proportional to a ratio between prediction accuracy and complexity. This means, for example, that models with high accuracy and a small number of parameters have a high NetScore value; on the contrary, models with moderate accuracy but high complexity have a small NetScore value. Furthermore, models with different accuracy, number of parameters, and MAC operations may have comparable values in terms of the NetScore metric. 
Finally, the relative impact of the components of the logarithmic ratio of the NetScore (namely, accuracy, architectural and computational complexities) are regulated by the exponential coefficients $\alpha, \beta$, and $\gamma$, which, in turn, allow us to adjust the weight of each factor according to its practical relevance for the application at hand. As an example, higher (than default) values can be assigned to $\beta$ and $\gamma$ whenever the NN model needs to be extremely simple in terms of computational and architectural complexities. On the other hand, if the model's accuracy is the most relevant algorithmic feature, then $\alpha$ can be set to a high value. In general, for the majority of the applications, it is possible to set $\alpha, \beta$, and $\gamma$ equal to $2,0.5$ and 0.5 , respectively [18]). This means that, from a general point of view, the relevance of the model's accuracy is usually higher than the (architectural and computational) complexity of the model itself.

\section{Related Work}

In Table 1, relevant literature references are summarized, which are commented in more detail below. As outlined in Section 1, the joint adoption of IoT and ML in the area of SA allows to simplify the maintenance of a greenhouse micro-climate, thus automatizing the control of its inner variables. Usually, this challenging and complex goal is pursued through three main steps. First, a WSN is deployed inside the greenhouse in order to collect and monitor a subset of relevant environmental parameters (namely, the greenhouse internal variables) $[7,9,11]$. Second, one or more control systems, based on devices that can control the greenhouse's actuators, are introduced in order to automatically maintain a proper internal climate $[12,13]$. Third, the internal variables' future trends are forecast with prediction techniques, based on ML algorithms, such as NNs [3,14-16,27,28].

In the context of greenhouse air temperature forecasting, NNs, due to their ability in learning patterns from data related to non-linear systems without an a priori knowledge of the system model [28], have become an extremely popular alternative to more consolidated techniques-e.g., physical methods based on mathematical theory and black-box approaches based on modern computational technology (e.g., Particle Swarm Optimization, PSO) [4].

As shown in Table 1, several algorithms to forecast the air temperature with NNbased models have been proposed. Although aiming at the same purpose, models in Table 1 differ in terms of adopted input variables, NN architecture, and data sources. Indeed, input parameters can include variables related to the surrounding environment external to the greenhouse, as well as environmental variables internal to the greenhouse, or a combination of them. Usually, the first group of variables cannot be controlled but influence the internal climate of the greenhouse: this is the case, for example, of external temperature, solar radiation, humidity, and wind speed $[3,14,15,27]$. In most cases, the second group of variables can instead be influenced by activating (or deactivating) some actuators installed inside the greenhouse: this is the case, for example, of internal air temperature and humidity, soil moisture, and $\mathrm{CO}_{2}[14,16,28]$. As outlined in Table 1 , the main types of NN architectures discussed in the literature, and achieving satisfactory prediction performance, are ANN, RNN, LSTM and Radial Basis Function (RBF) networks.

Considering the principles on the basis of their designs, it is possible to conceptually separate literature models into the following categories: (i) time series-oriented approaches, (ii) "pure" ML approaches, and (iii) hybrid approaches. In detail, the first type of approaches solves the task of predicting future air temperature values as a time series forecasting problem, leveraging the following features characterizing time series data, i.e., data which are periodically sampled and have a time reference (as sensor data): trends, seasonality, and correlation between samples which are close in time. The time series peculiarity of having, in most cases, temporally-close data linked by a relation, can be successfully discovered by NN models, such as RNNs [27] and LSTM networks [28], and exploited in order to achieve prediction performance better than other types of NN architectures. The use of data coming from other data sources, without taking into account the relation existing between temporally-close data of the same time series, can also be 
valuable in order to forecast air temperature. These sources can include data related to external or internal variables to the greenhouse, correlated to the air temperature values to be predicted. With this approach, the use of RBF networks [15,16] and ANNs [29] have been very successful in air temperature forecasting. To conclude, the two approaches can be integrated leading to a hybrid approach, selecting the $\mathrm{NN}$ architecture which best suits the prediction problem at hand [3].

The authors now comment briefly on the limitations of existing literature and on the steps forward proposed in the current paper. In [3], an air temperature forecasting model based on an ANN, predicting air temperature with a RMSE of $2.5 \div 3.0^{\circ} \mathrm{C}$, has been presented. Better results, in terms of RMSE, have been achieved with the same type of $\mathrm{NN}$ architecture (namely, ANN) in [14,19], reaching a RMSE equal to $0.839^{\circ} \mathrm{C}$ and $1.50{ }^{\circ} \mathrm{C}$, respectively. These results can be eventually justified by the fact that the last two models have been trained with a wider data set (in other words, a data set with a number of samples 4 or 64 times greater with respect to the one available in [3]). In [27], a RNN-based model has been built using a data set of comparable size to [3] but achieving a better RMSE (equal to $0.865^{\circ} \mathrm{C}$ ) than $[19,27]$, but lower than [14]. Moreover, RBF networks have been adopted in [16] to build a model which outperforms all the above-mentioned papers in terms of RMSE. Unlike these literature works, in which the forecasting model designs take into account only one type of NN architecture, in $[15,28]$, more than one type of NN architecture have been compared in order to perform a more comprehensive analysis. The prediction performance of the models presented in $[15,28]$ is comparable with that in $[14,27]$, better than that in [3,19], but lower than that in [16].

The above-cited works (also outlined in Table 1) do not provide any detail concerning the computational and architectural complexities of their proposed models. Instead, such complexities are key factors while designing IoT applications in which NN-based algorithms are deployed on embedded devices (usually located on the network's edge). Indeed, since most literature works-showing remarkable results in the air temperatures forecasting (e.g., with a RMSE value lower than $1^{\circ} \mathrm{C}$ )—are intended to be executed in the Cloud and not on IoT (edge) devices, no discussion concerning the proposed model's complexity is provided, as this aspect is not a limitation. However, since the diffusion of EdgeAI (due to its significant advantages) is rapidly growing, taking into account the complexity, while deploying new algorithms for IoT applications (running on edge devices), is extremely relevant. In the above-cited works, this aspect has not been exhaustively discussed in the context of forecasting air temperature within greenhouses. Filling this literature gap is one of the aims of the current paper. 
Table 1. Representative literature papers in the context of air temperature forecasting inside greenhouses through the usage of NNs.

\begin{tabular}{|c|c|c|c|c|c|c|c|c|c|}
\hline \multirow[b]{2}{*}{ Ref. } & \multicolumn{3}{|c|}{ NN Model } & \multicolumn{3}{|c|}{ Performances (on Test Set) } & \multicolumn{3}{|c|}{ Data Set Details } \\
\hline & Input Variables & $\begin{array}{l}\text { Architectural } \\
\text { Type }\end{array}$ & $\begin{array}{c}\text { Training } \\
\text { Algorithm }\end{array}$ & $\operatorname{RMSE}\left({ }^{\circ} \mathrm{C}\right)$ & MAPE (\%) & $\mathbf{R}^{2}$ & $\begin{array}{c}\text { Size } \\
\text { (Samples No) }\end{array}$ & $\begin{array}{l}\text { Collection } \\
\text { Interval }\end{array}$ & $\begin{array}{l}\text { Sampling } \\
\text { Interval }\end{array}$ \\
\hline$[3]$ & $\begin{array}{l}\text { External temperature and } \\
\text { solar radiation, wind speed, } \\
\text { heater temperature, } \\
\text { datetime reference }\end{array}$ & ANN & $\mathrm{BP}, \mathrm{CGA}$ & $2.5-3.0$ & $\mathrm{~N} / \mathrm{A}$ & $\mathrm{N} / \mathrm{A}$ & 1368 & $\approx 2$ months & $1 \mathrm{~h}$ \\
\hline [14] & $\begin{array}{l}\text { Internal solar radiation, air } \\
\text { temperature and humidity, and } \\
\text { soil moisture, } \mathrm{CO}_{2}, \\
\text { atmospheric pressure, } \\
\text { datetime reference }\end{array}$ & ANN & $\mathrm{BP}$ & 0.839 & $\mathrm{~N} / \mathrm{A}$ & 0.977 & $\approx 87,408$ & 19 months & $10 \mathrm{~min}$ \\
\hline [15] & $\begin{array}{l}\text { External solar radiation and } \\
\text { temperature, wind speed }\end{array}$ & ANN, RBF & $\mathrm{BP}$ & $\begin{array}{l}0.20 \pm 0.02 \\
0.13 \pm 0.01\end{array}$ & $\begin{array}{l}0.93 \pm 0.10 \\
0.59 \pm 0.07\end{array}$ & $\begin{array}{l}0.76 \pm 0.05 \\
0.89 \pm 0.03\end{array}$ & $\mathrm{~N} / \mathrm{A}$ & $\mathrm{N} / \mathrm{A}$ & $\mathrm{N} / \mathrm{A}$ \\
\hline [16] & $\begin{array}{l}\text { External solar radiation, heater } \\
\text { temperature, internal air } \\
\text { temperature and humidity, } \\
\text { wind speed, history of } \\
\text { actuators, shadow screen }\end{array}$ & RBF & $\mathrm{BP}, \mathrm{LM}$ & 0.0019 & $\mathrm{~N} / \mathrm{A}$ & $\mathrm{N} / \mathrm{A}$ & 1728 & 12 days & $10 \mathrm{~min}$ \\
\hline [19] & $\begin{array}{c}\text { External apparent temperature, } \\
\text { dew point, air humidity, air } \\
\text { temperature and UV index, } \\
\text { datetime reference }\end{array}$ & ANN & $\mathrm{BP}$ & 1.50 & 4.91 & 0.965 & 5346 & 10 months & $1 \mathrm{~h}$ \\
\hline [28] & $\begin{array}{l}\text { External temperature, solar } \\
\text { radiation and humidity, wind } \\
\text { speed and direction, history } \\
\text { of actuators }\end{array}$ & $\begin{array}{l}\text { ANN, } \\
\text { RNN-LSTM, } \\
\text { NARX }\end{array}$ & $\mathrm{BP}$ & $\begin{array}{l}0.89-0.94 \\
0.45-0.71 \\
0.52-1.32\end{array}$ & $\mathrm{~N} / \mathrm{A}$ & $\begin{array}{l}0.94,0.96-0.97 \\
0.86-0.96\end{array}$ & $\approx 470,000$ & 1 year & $\begin{array}{c}5,10,15,20,25, \\
30 \mathrm{~min}\end{array}$ \\
\hline [27] & $\begin{array}{l}\text { Internal air and soil } \\
\text { temperature, internal solar } \\
\text { radiation, humidity and } \mathrm{CO}_{2}\end{array}$ & RNN & $\mathrm{BP}$ & 0.865 & 1.7 & 0.925 & 1152 & 8 days & $10 \mathrm{~min}$ \\
\hline
\end{tabular}


To conclude, in a previous work [19], the authors have discussed the design of an edge device-friendly air temperature forecasting model following the pure ML approach (the second approach previously introduced). More precisely, in order to forecast future values of air temperature inside a greenhouse in a demonstrator of the H2020 project AFarCloud [30], the authors have selected the external weather conditions as input variables for the model. Moreover, the authors have implemented an algorithm which, due to its low computational and architectural complexities, can be easily run by an edge device (i.e., the Smart GW) and can predict future air temperatures with performance results comparable to the literature. As anticipated at the end of Section 1, another goal of this paper is to experimentally investigate a novel time series-based approach in a comparative way with respect to [19] and existing literature approaches, including a comprehensive analysis of the prediction performance in relative terms with respect to the (computational and architectural) complexity of the developed model. In particular, the authors will show that their model, even with limited complexity, incurs minimal (or no) performance degradation with respect to Cloud-oriented approaches.

\section{Methodology}

As outlined in Section 1, the aim of this paper is three-fold. First, the authors want to evaluate an alternative approach to solve the ML-oriented task presented in [19], namely the deployment of an "edge device-friendly" air temperature forecasting model (intended to be run on a Smart GW). Instead of the weather conditions external to the greenhouse, the NN-based model deployed with the novel approach (i.e., time series-oriented, as discussed in Section 3) takes as input variables only air temperature values collected inside the greenhouse. Three architectural types of NN are considered: ANN, RNN, and LSTM. The authors analyze the impact, on the prediction performance of the selected three architectures, of two design parameters: (i) the number of model's input variables (or, in other terms, the size of the data sliding window used by the model), denoted as $S W$, and (ii) their sampling period, denoted as $T_{\text {samp }}$ (dimension: [min]). Second, the model presented in [19] has been re-trained with data collected from August 2019 to the end of November 2020, to make the data set coherent with that available for the new approach (in [19], the data set was composed by data collected from August 2019 to the end of May 2020). Third, the experimental performances of the proposed models have been compared with those outlined in Table 1.

From a methodological point of view, the following steps are undertaken.

1. Relevant air temperature data, measured with sensors inside a greenhouse associated with an Italian demonstrator of the H2020 project AFarCloud [30], are collected and processed to remove outliers and spurious data (Section 4.1).

2. The greenhouse indoor temperature sensor data collected with a sampling period $T_{\text {samp }}=10 \mathrm{~min}$ are arranged in a time series. Furthermore, from this original time series, six additional time series are derived downsampling the first time series with longer sampling periods (Section 4.2).

3. The number of input variables of the model $S W$ and the sampling period $T_{\text {samp }}$ are defined as the two design parameters. Moreover, $T_{\text {samp }}$ is reintegrated as the prediction time horizon; in fact, the predicted temperature value is the one corresponding to the next temperature value after the most recent one of the sliding window: this samples is, by construction, $T_{\text {samp }}$ ahead. Furthermore, a proper set of values related to these parameters is selected for testing purposes (Section 4.3).

4. Starting from the collected sensor data and according to the number of parameters values to be tested, multiple data sets are created. Furthermore, each data set is split into training and test subsets (Section 4.4). 
5. Three NN architectures, based on an ANN, a RNN, and a LSTM, are introduced and trained with the data sets resulting from the previous steps (Section 4.5).

6. The NN model presented in [19] is re-trained with a significantly larger data setincluding data from 6 more months (Section 4.6).

7. All models are evaluated on the test subsets and their performances are compared in terms of RMSE, MAPE, $\mathrm{R}^{2}$, and NetScore (Section 5).

8. Finally, the best three models (among a total of 210) on the considered engineered data sets (step 4) are performance-wise compared with relevant literature approaches (Section 5).

\subsection{Data Collection and Cleaning}

Sensor data related to air temperatures have been gathered inside the greenhouse of an Italian farm, denoted as Podere Campàz [31], through the LoRaFarM platform, a Farm-as-a-Service (FaaS) architecture proposed in [8]. In detail, air temperature values have been measured with a sampling interval of $10 \mathrm{~min}$, for a time period of 16 months-from the beginning of August 2019 to the end of November 2020. The distribution of collected data, over the 16-month time period, is shown in Figure 4: in each month, the authors indicate the average (over the month) number of data collected daily.

Analyzing the data distribution shown in Figure 4, it can be seen that data have been irregularly collected across the months of the collection period. This is due to various reasons: for example, during December 2019, March 2020, and September 2020, the data irregularity was caused by a temporary Internet connectivity loss, which prevented collected data from being forwarded, through the LoRaFarM platform, to a storage repository placed in the Cloud.

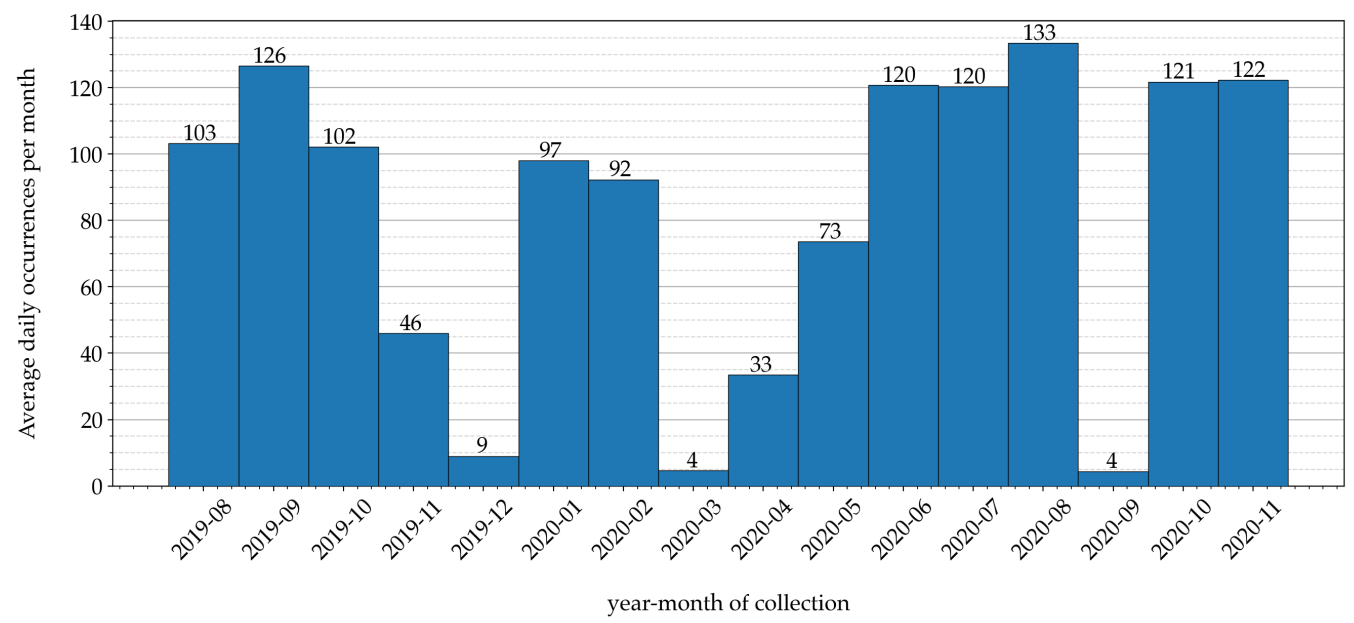

Figure 4. Sensor data collected during a 16-month time period: in each month, the average daily number of collected samples (obtained as the ratio between the number of gathered samples per month normalized and the number of days of the month) is shown.

On the other hand, the samples' distribution over actual collection daily hours has an almost perfect uniform trend, meaning that the number of data collected during different hours of the day in the 16-month time period is approximately the same at each hour. Indeed, the overall amount of "raw" data gathered during this stage correspond to $N_{\text {samp }}=40,033$ samples, with the number of samples collected per hours varying from a minimum of 1649 samples to a maximum of 1694 samples, with an average value of 1668 samples and a standard deviation of approximately 11 samples. 


\subsection{Engineering Time Series from Sensor Data}

As mentioned in Section 4, sensor data were collected with a (real) sampling period $T_{\text {samp }}=T_{0}=10 \mathrm{~min}$. The corresponding time series, describing the measured air temperature inside the greenhouse during the considered period of 16 months, is denoted as $\left\{z_{k}^{\left(T_{0}\right)}\right\}_{k=1}^{N_{\text {tot }}}$, where $k$ corresponds to the time instant $k T_{0}$-denoting $T_{0}$ (with $k=1$ ) as the instant of collection of the first sample and $N_{\text {tot }} T_{0}$ (with $k=N_{\text {tot }}$ ) as the instant of collection of the last sample-or, with a more compact notation, simply $\left\{z_{k}^{\left(T_{0}\right)}\right\}$. More precisely, denoting the temperature in the greenhouse as $z(t)$, the time series' sample $z_{k}^{\left(T_{0}\right)}=z\left(k T_{0}\right)$ corresponds to the air temperature measured at time instant $t=k T_{0}$. As an additional clarification, in this paper a deterministic temperature signal $z(t)$ has been considered, as it refers to the specific real data collected from sensors. In order to generalize this approach, the air temperature should be modeled as a stochastic process $Z(t)$. However, this goes beyond the scope of this paper. In Figure 5, an illustrative representation of the first 18 samples of the time series $\left\{z_{k}^{\left(T_{0}\right)}\right\}$ (black dots), denoted as $\left\{z_{k}\right\}$ for simplicity, is shown.

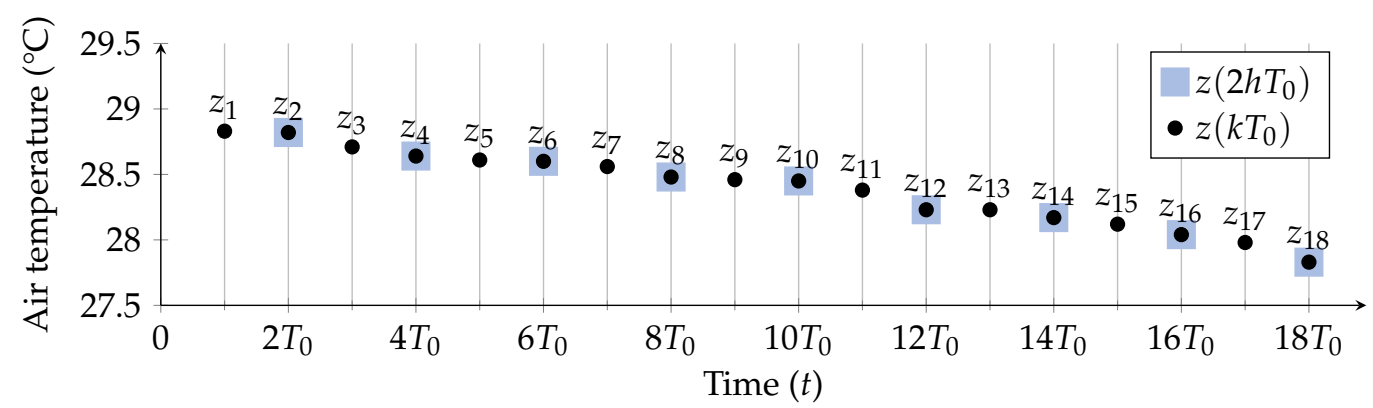

Figure 5. Illustrative representation of the first 18 samples $\left\{z_{k}^{\left(T_{0}\right)}=z\left(k T_{0}\right)\right\}_{k=1}^{18}$ (black dots) and of the first 9 samples $\left\{z_{h}^{\left(2 T_{0}\right)}=z\left(2 h T_{0}\right)\right\}_{h=1}^{9}$ (black dots over violet squares), obtained downsampling $\left\{z_{k}^{\left(T_{0}\right)}\right\}$ with a factor equal to 2 .

The authors remark that, because of the methodology selected to gather sensor data inside the greenhouse, some samples of the time series $\left\{z_{k}^{\left(T_{0}\right)}\right\}$ may not be available-i.e., the LoRaFarM platform may suffer from temporary lack of Internet connectivity and, thus, lose some sensor data. Hence, this means that there exist $N_{\text {lost }}$ instants of time $\left(\hat{k}_{1} T_{0}, \ldots, \hat{k}_{N_{\text {lost }}} T_{0}\right)$ at which the values of air temperature $\left\{z_{\hat{k}_{i}}^{\left(T_{0}\right)}\right\}_{i=1}^{N_{\text {lost }}}$ are not available-for example, one can set $z_{\hat{k}_{i}}^{\left(T_{0}\right)}=\mathrm{NaN}$. Moreover, for this reason, the overall number of samples in the time series, denoted as $N_{\text {samp }}$, is smaller than the total number of samples $N_{\text {tot }}$ ideally collected in the considered 16 month time interval with a sampling period equal to $T_{0}=10 \mathrm{~min}$ and no data loss-more precisely, $N_{\text {tot }}=N_{\text {samp }}+N_{\text {lost }}$.

Focusing on downsampling, it is useful to reduce the number of samples in a time series in the context of time series' forecasting and analysis, indicating with $\lambda$ the downsampling factor, the downsampled time series can be expressed as follows:

$$
\left\{z_{h}^{\left(\lambda T_{0}\right)}=z\left(\lambda h T_{0}\right)\right\}_{h}=\left\{z_{\lambda h}^{\left(T_{0}\right)}\right\}_{\lambda h} \quad h=1,2, \ldots
$$

To this end, in Figure 5 the downsampled version (with $\lambda=2$ ) of the original time series is shown, with the corresponding samples identified by circles with squares.

In order to evaluate how the sampling period $T_{\text {samp }}$ influences the prediction performance of the model, 6 additional time series are built downsampling the original time series $\left\{z_{k}^{\left(T_{0}\right)}\right\}$ with $T_{\text {samp }}$ equal to $2 T_{0}=20 \mathrm{~min}, 3 T_{0}=30 \mathrm{~min}, 4 T_{0}=40 \mathrm{~min}, 5 T_{0}=50 \mathrm{~min}$, $6 T_{0}=60 \mathrm{~min}$, and $12 T_{0}=120 \mathrm{~min}$, i.e., by setting the downsampling factor $\lambda$ to 2, 3, 4, 5,6 , and 12 , respectively. The corresponding time series are denoted as $\left\{z_{k}^{\left(2 T_{0}\right)}\right\},\left\{z_{k}^{\left(3 T_{0}\right)}\right\}$, $\left\{z_{k}^{\left(4 T_{0}\right)}\right\},\left\{z_{k}^{\left(5 T_{0}\right)}\right\},\left\{z_{k}^{\left(6 T_{0}\right)}\right\},\left\{z_{k}^{\left(12 T_{0}\right)}\right\}$, respectively. 
All the created time series have been used to build a total of 70 different data sets (i.e., 10 data sets for every time series, with a different number of input variables each), as detailed in Section 4.4. Considering these time series allows to determine the best sampling period for the phenomenon of interest (inner air temperature).

\subsection{Sliding Window-Based Prediction}

As described in Section 4, the prediction approach is based on the use of a sliding window. More precisely, the authors consider SW consecutive samples of the target time series, with sampling interval $T_{\text {samp }}$, to predict the value of the next sample. With reference to Figure 6, at epoch $k$ :

$$
\hat{z}_{k+1}^{\left(T_{\text {samp }}\right)}=\hat{z}_{k+1}^{\left(T_{\text {samp }}\right)}\left(z_{k-S W+1}^{\left(T_{\text {samp }}\right)}, \ldots, z_{k}^{\left(T_{\text {samp }}\right)}\right)
$$

where $\hat{z}_{k+1}^{\left(T_{\text {samp }}\right)}$ corresponds to the predicted value of the (true) sample $z_{k+1}^{\left(T_{\text {samp }}\right)}$ and, at the right side, the dependency from the previous samples is highlighted.

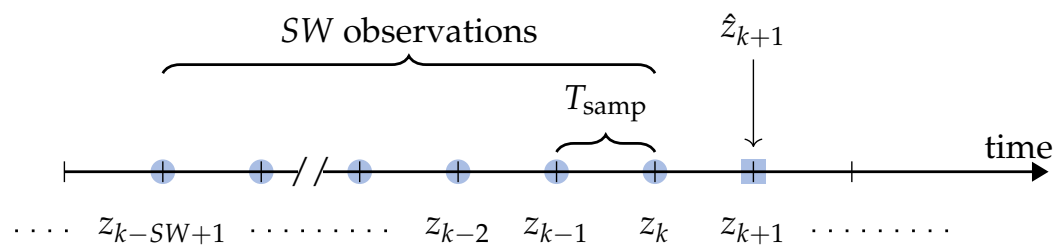

Figure 6. Sliding window-based prediction at epoch $k$ : the $S W$ observations $\left\{z_{k-i+1}\right\}_{i=1}^{S W}$ are used to predict the value $z_{k+1}$, denoted as $\hat{z}_{k+1}$ - the superscript ${ }^{\left(T_{\text {samp }}\right)}$ is omitted for simplicity.

Since the parameters $S W$ and $T_{\text {samp }}$ influence the prediction performance of the model, they need to be optimized. For this reason, different combinations of $S W$ and $T_{\text {samp }}$ are considered, comparing the corresponding prediction performance. In detail, 10 values of $S W$ (namely, from 1 to 10 samples) and, as described in Section 4.2, 7 values for $T_{\text {samp }}$ (namely, 10, 20, 30, 40, 50, 60 and $120 \mathrm{~min}$ ) are considered.

For the sake of clarity, it is noteworthy to remark that, since $S W$ corresponds to the input size of a model (the parameter $\ell$ in Figure 2 for a neuron of the input layer), at the input of the model there is a $S W$-dimensional vector of $S W$ features corresponding to air temperature values sampled with a fixed period $T_{\text {samp }}$ and, thus, coming from the time series $\left\{z_{k}^{\left(T_{\text {samp }}\right)}\right\}$ built in the previous stage. As an example, a model built selecting $S W=4$ and $T_{\text {samp }}=10 \mathrm{~min}$ is fed with input vectors having 4 features corresponding to 4 consecutive air temperature samples collected every $T_{\text {samp }}=10 \mathrm{~min}$.

\subsection{Data Pre-Processing and Data Sets Creation}

Since the authors want to evaluate the prediction performance of the model adopting different combinations of $\left\{S W, T_{\text {samp }}\right\}$ pairs, multiple data sets to train and test the algorithm can be engineered. More precisely, 70 data sets have been created, each one associated with a specific $\left\{S W, T_{\text {samp }}\right\}$ pair, where $S W \in\{1,2, \ldots, 10\}$ and $T_{\text {samp }} \in$ $\{10,20,30,40,50,60,120\} \mathrm{min}$. In detail, using a compact notation, the authors denote as $\mathcal{D}_{S W}^{\left(T_{\text {samp }}\right)}$ the data set obtained with the corresponding values of $T_{\text {samp }}$ and $S W$. Each entry of the data set is a vector $\underline{d}(k)$ of true temperature values defined as follows:

$$
\underline{d}(k) \triangleq\left(z_{k-S W+1}^{\left(T_{\mathrm{samp}}\right)}, \ldots, z_{k}^{\left(T_{\mathrm{samp}}\right)}, z_{k+1}^{\left(T_{\mathrm{samp}}\right)}\right) .
$$

In a compact notation, one can write:

$$
\underline{d}(k)=(\underline{x}(k), y(k))
$$


where

$$
\underline{x}(k) \triangleq\left(x_{1}(k), \ldots, x_{S W}(k)\right)
$$

with

$$
x_{i}(k)=z_{k-i+1}^{\left(T_{\text {samp }}\right)} \quad i=1, \ldots, S W
$$

is the vector of the input variable of the model and $y(k)=z_{k+1}^{\left(T_{\text {samp }}\right)}$ is the true temperature value which has to be estimated by the output of the NN based on $\underline{x}(k)$. In Figure 7, the generic entry $\underline{d}(k)$ of $\mathcal{D}_{S W}^{\left(T_{\text {samp }}\right)}$, for $k>S W$, is shown.

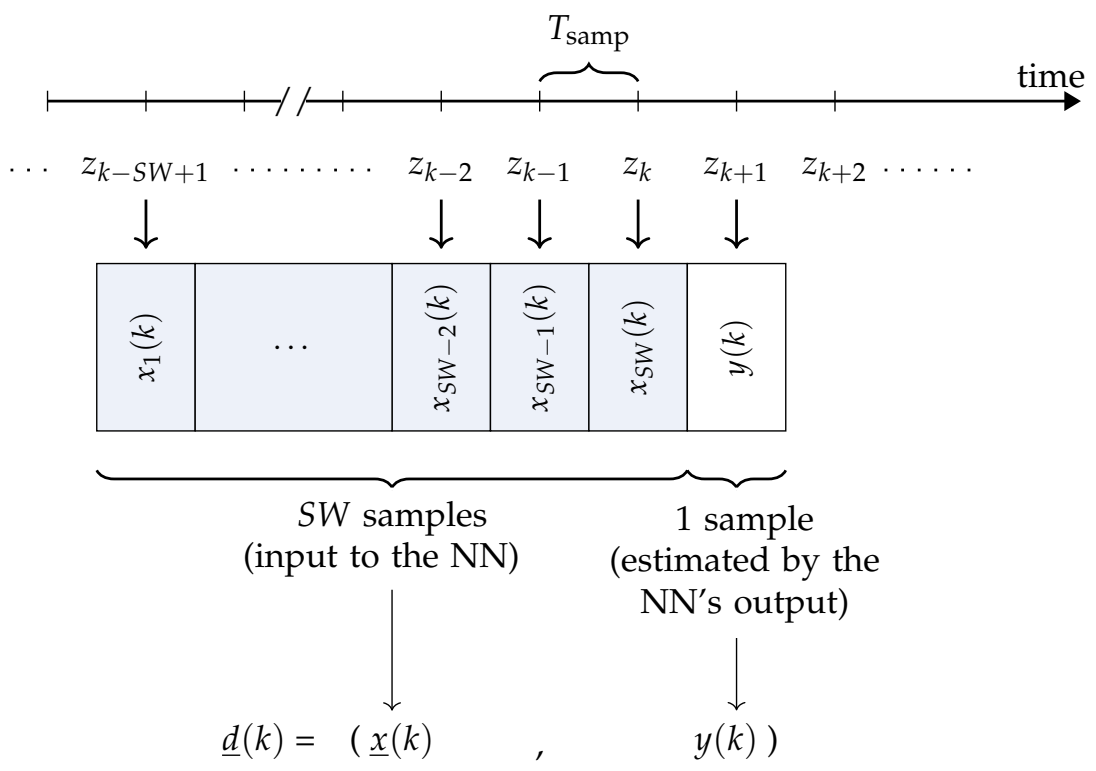

Figure 7. The $k$-th sample $\underline{d}(k)$ in the data set $\mathcal{D}_{S W}^{\left(T_{\text {samp }}\right)}$ is composed of $S W$ values of air temperatures (composing the $S W$-dimensional vector $\underline{x}(k)$ of input variables) and of an output variable $y(k)$ (corresponding to the air temperature at epoch $k+1$, which has to be forecast starting from $\underline{x}(k)$ ).

As a side note, entries with one or more NaN values-corresponding to missing air temperature values for specific instants of time in the original time series, as explained in Section 4.2-have been discarded and not included in the data sets. Then, each data set is split (randomly) into a training subset and a test subset with a ratio $3: 1$.

More details on the created data sets, in terms of number of samples in the set (divided among training subset and test subset) and values of $S W$ and $T_{\text {samp }}$, can be found in Table 2. 
Table 2. Details concerning the engineered data sets, in terms of number of samples, $S W$ and $T_{\text {samp. }}$.

\begin{tabular}{|c|c|c|c|c|c|c|c|c|c|c|c|}
\hline Data Set & $\begin{array}{c}T_{\text {samp }} \\
{[\text { [min] }}\end{array}$ & $\begin{array}{c}S W \\
\text { [Samples] }\end{array}$ & $\begin{array}{c}\text { Size } \\
\text { [Samples] }\end{array}$ & $\begin{array}{l}\text { Training Subset } \\
\text { Size [Samples] }\end{array}$ & $\begin{array}{l}\text { Test Subset Size } \\
\text { [Samples] }\end{array}$ & Data Set & $\begin{array}{c}T_{\text {samp }} \\
{[\text { [min] }}\end{array}$ & $\begin{array}{c}S W \\
\text { [Samples] }\end{array}$ & $\begin{array}{c}\text { Size } \\
\text { [Samples] }\end{array}$ & $\begin{array}{l}\text { Training Subset } \\
\text { Size [Samples] }\end{array}$ & $\begin{array}{l}\text { Test Subset Size } \\
\text { [Samples] }\end{array}$ \\
\hline \multirow{5}{*}{$\begin{array}{c}\mathcal{D}_{1}^{(10)} \\
\mathcal{D}_{2}^{(10)} \\
\mathcal{D}_{4}^{(10)} \\
\mathcal{D}_{6}^{(10)} \\
\mathcal{D}_{8}^{(10)} \\
\end{array}$} & 10 & 1 & 36,330 & 27,248 & 9082 & \multirow{5}{*}{$\begin{array}{l}\mathcal{D}_{10}^{(10)} \\
\mathcal{D}_{3}^{(10)} \\
\mathcal{D}_{5}^{(10)} \\
\mathcal{D}_{7}^{(10)} \\
\mathcal{D}_{9}^{(10)} \\
\end{array}$} & 10 & 10 & 32,696 & 24,522 & 8174 \\
\hline & 10 & 2 & 35,828 & 26,871 & 8957 & & 10 & 3 & 35,363 & 26,523 & 8840 \\
\hline & 10 & 4 & 34,923 & 26,193 & 8730 & & 10 & 5 & 34,512 & 25,884 & 8628 \\
\hline & 10 & 6 & 34,118 & 25,589 & 8529 & & 10 & 7 & 33,734 & 25,301 & 8433 \\
\hline & 10 & 8 & 33,366 & 25,025 & 8341 & & 10 & 9 & 33,020 & 24,765 & 8255 \\
\hline \multirow{5}{*}{$\begin{array}{l}\mathcal{D}_{1}^{(120)} \\
\mathcal{D}_{2}^{(120)} \\
\mathcal{D}_{4}^{(120)} \\
\mathcal{D}_{6}^{(120)} \\
\mathcal{D}_{8}^{(120)} \\
\end{array}$} & 120 & 1 & 2985 & 2239 & 746 & \multirow{5}{*}{$\begin{array}{l}\mathcal{D}_{10}^{(120)} \\
\mathcal{D}_{3}^{(120)} \\
\mathcal{D}_{5}^{(120)} \\
\mathcal{D}_{7}^{(120)} \\
\mathcal{D}_{9}^{(120)} \\
\end{array}$} & 120 & 10 & 2457 & 1843 & 614 \\
\hline & 120 & 2 & 2912 & 2184 & 728 & & 120 & 3 & 2843 & 2133 & 710 \\
\hline & 120 & 4 & 2781 & 2086 & 695 & & 120 & 5 & 2723 & 2043 & 680 \\
\hline & 120 & 6 & 2666 & 2000 & 666 & & 120 & 7 & 2611 & 1959 & 652 \\
\hline & 120 & 8 & 2558 & 1919 & 639 & & 120 & 9 & 2507 & 1881 & 626 \\
\hline \multirow{5}{*}{$\begin{array}{l}\mathcal{D}_{1}^{(20)} \\
\mathcal{D}_{2}^{(20)} \\
\mathcal{D}_{4}^{(20)} \\
\mathcal{D}_{6}^{(20)} \\
\mathcal{D}_{8}^{(20)} \\
\end{array}$} & 20 & 1 & 18,102 & 13,577 & 4525 & \multirow{5}{*}{$\begin{array}{l}\mathcal{D}_{10}^{(20)} \\
\mathcal{D}_{3}^{(20)} \\
\mathcal{D}_{5}^{(20)} \\
\mathcal{D}_{7}^{(20)} \\
\mathcal{D}_{9}^{(20)} \\
\end{array}$} & 20 & 10 & 16,006 & 12,005 & 4001 \\
\hline & 20 & 2 & 17,812 & 13,359 & 4453 & & 20 & 3 & 17,539 & 13,155 & 4384 \\
\hline & 20 & 4 & 17,286 & 12,965 & 4321 & & 20 & 5 & 17,050 & 12,788 & 4262 \\
\hline & 20 & 6 & 16,822 & 12,617 & 4205 & & 20 & 7 & 16,605 & 12,454 & 4151 \\
\hline & 20 & 8 & 16,399 & 12,300 & 4099 & & 20 & 9 & 16,196 & 12,147 & 4049 \\
\hline \multirow{5}{*}{$\begin{array}{l}\mathcal{D}_{1}^{(30)} \\
\mathcal{D}_{2}^{(30)} \\
\mathcal{D}_{4}^{(30)} \\
\mathcal{D}_{6}^{(30)} \\
\mathcal{D}_{8}^{(30)} \\
\end{array}$} & 30 & 1 & 12,067 & 9051 & 3016 & \multirow{5}{*}{$\begin{array}{l}\mathcal{D}_{10}^{(30)} \\
\mathcal{D}_{3}^{(30)} \\
\mathcal{D}_{5}^{(30)} \\
\mathcal{D}_{7}^{(30)} \\
\mathcal{D}_{9}^{(30)} \\
\end{array}$} & 30 & 10 & 10,618 & 7964 & 2654 \\
\hline & 30 & 2 & 11,862 & 8897 & 2965 & & 30 & 3 & 11,678 & 8759 & 2919 \\
\hline & 30 & 4 & 11,504 & 8628 & 2876 & & 30 & 5 & 11,341 & 8506 & 2835 \\
\hline & 30 & 6 & 11184 & 8388 & 2796 & & 30 & 7 & 11,030 & 8273 & 2757 \\
\hline & 30 & 8 & 10,885 & 8164 & 2721 & & 30 & 9 & 10,749 & 8062 & 2687 \\
\hline \multirow{5}{*}{$\begin{array}{l}\mathcal{D}_{1}^{(40)} \\
\mathcal{D}_{2}^{(40)} \\
\mathcal{D}_{4}^{(40)} \\
\mathcal{D}_{6}^{(40)} \\
\mathcal{D}_{8}^{(40)} \\
\end{array}$} & 40 & 1 & 9008 & 6756 & 2252 & \multirow{5}{*}{$\begin{array}{l}\mathcal{D}_{10}^{(40)} \\
\mathcal{D}_{3}^{(40)} \\
\mathcal{D}_{5}^{(40)} \\
\mathcal{D}_{7}^{(40)} \\
\mathcal{D}_{9}^{(40)} \\
\end{array}$} & 40 & 10 & 7690 & 5768 & 1922 \\
\hline & 40 & 2 & 8829 & 6622 & 2207 & & 40 & 3 & 8656 & 6492 & 2164 \\
\hline & 40 & 4 & 8495 & 6372 & 2123 & & 40 & 5 & 8341 & 6256 & 2085 \\
\hline & 40 & 6 & 8196 & 6147 & 2049 & & 40 & 7 & 8062 & 6047 & 2015 \\
\hline & 40 & 8 & 7931 & 5949 & 1982 & & 40 & 9 & 7806 & 5855 & 1951 \\
\hline \multirow{5}{*}{$\begin{array}{l}\mathcal{D}_{1}^{(50)} \\
\mathcal{D}_{2}^{(50)} \\
\mathcal{D}_{4}^{(50)} \\
\mathcal{D}_{6}^{(50)} \\
\mathcal{D}_{8}^{(50)}\end{array}$} & 50 & 1 & 7220 & 5415 & 1805 & \multirow{5}{*}{$\begin{array}{l}\mathcal{D}_{10}^{(50)} \\
\mathcal{D}_{3}^{(50)} \\
\mathcal{D}_{5}^{(50)} \\
\mathcal{D}_{7}^{(50)} \\
\mathcal{D}_{9}^{(50)}\end{array}$} & 50 & 10 & 6185 & 4639 & 1546 \\
\hline & 50 & 2 & 7079 & 5310 & 1769 & & 50 & 3 & 6944 & 5208 & 1736 \\
\hline & 50 & 4 & 6816 & 5112 & 1704 & & 50 & 5 & 6695 & 5022 & 1673 \\
\hline & 50 & 6 & 6584 & 4938 & 1646 & & 50 & 7 & 6479 & 4860 & 1619 \\
\hline & 50 & 8 & 6376 & 4782 & 1594 & & 50 & 9 & 6280 & 4710 & 1570 \\
\hline \multirow{5}{*}{$\begin{array}{l}\mathcal{D}_{1}^{(60)} \\
\mathcal{D}_{2}^{(60)} \\
\mathcal{D}_{4}^{(60)} \\
\mathcal{D}_{6}^{(60)} \\
\mathcal{D}_{8}^{(60)}\end{array}$} & 60 & 1 & 6006 & 4505 & 1501 & \multirow{5}{*}{$\begin{array}{l}\mathcal{D}_{10}^{(60)} \\
\mathcal{D}_{3}^{(60)} \\
\mathcal{D}_{5}^{(60)} \\
\mathcal{D}_{7}^{(60)} \\
\mathcal{D}_{9}^{(60)}\end{array}$} & 60 & 10 & 5146 & 3860 & 1286 \\
\hline & 60 & 2 & 5886 & 4415 & 1471 & & 60 & 3 & 5772 & 4329 & 1443 \\
\hline & 60 & 4 & 5668 & 4251 & 1417 & & 60 & 5 & 5570 & 4178 & 1392 \\
\hline & 60 & 6 & 5478 & 4109 & 1369 & & 60 & 7 & 5389 & 4042 & 1347 \\
\hline & 60 & 8 & 5306 & 3980 & 1326 & & 60 & 9 & 5224 & 3918 & 1306 \\
\hline
\end{tabular}

\subsection{Models Training}

The NN models evaluated in this paper, based on LSTMs, RNNs, and ANNs, are shown in Figure 8a, Figure 8b and Figure 8c, respectively. As can be observed, these models share a similar structure, in terms of number of layers and neurons per layer, with the exception of the first hidden layer, which is not feed-forward and is composed of LSTM or RNN cells for the LSTM-based and the RNN-based models, respectively, while it is feed-forward for the ANN-based model. Moreover, as can be expected, the number of neurons in the input layer may vary from 1 to 10, according to the adopted values of $S W$.

Each model has been trained on the 70 data sets engineered in the previous stages (more precisely, on training subsets obtained from these data sets) using the BP algorithm [23] and considering the RMSE as loss function. From a practical point of view, Python v3.8.6 and the Keras framework v2.4.3 [32] have been used. Moreover, with regard to the other NN's learning parameters, the following values have been set: learning rate $=0.002$, batch size $=$ 20 , and number of epochs $=20$. In order to perform the fairest possible comparison among these NN models, the values of these parameters have been kept fixed for the three models. In all cases, the number of hidden layers and the number of neurons of the hidden layers are kept fixed as well in all NN models: they are set to 3 and to $32 / 8 / 3$, respectively. 


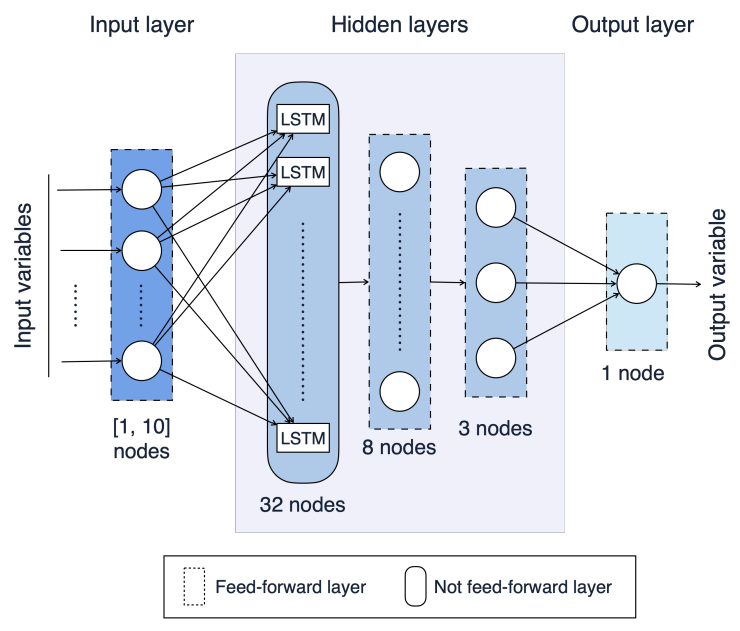

(a)

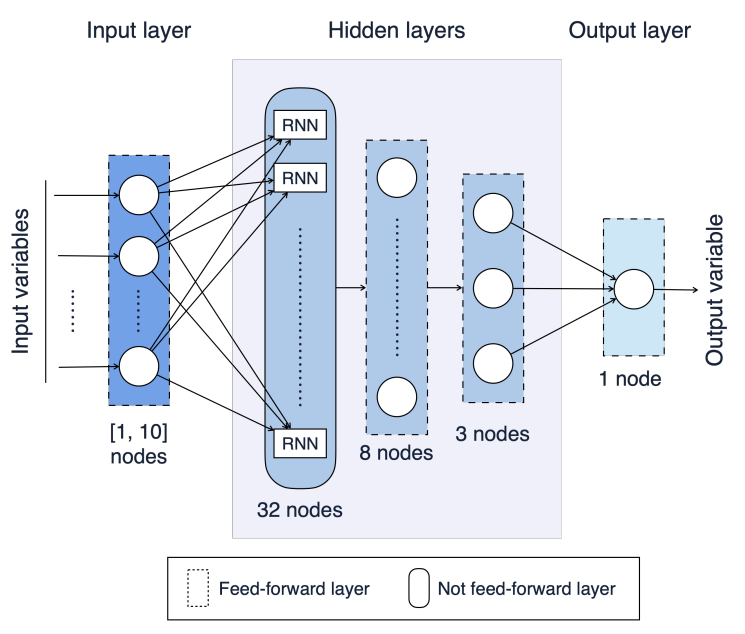

(b)

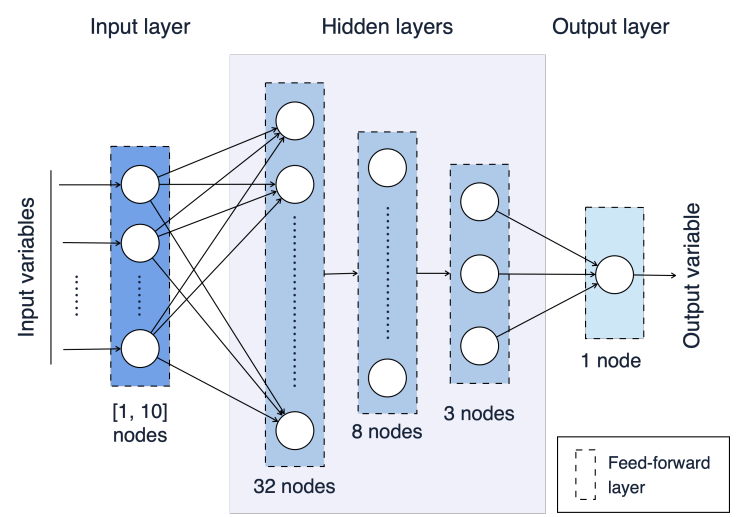

(c)

Figure 8. NN models evaluated in this paper: (a) LSTM-based; (b) RNN-based; (c) ANN-based.

\section{6. "Old Model" Re-Training}

In order to fairly compare the novel models proposed in this paper with the model presented in [19], the latter (referred in the following as "old model") has been re-trained with additional new data, namely, meteorological data and sensor data (related to air temperatures) gathered between the beginning of August 2019 and the end of November 2020. As a side note, the additional data (collected in 6 more months with regard to the time interval considered in [19]) allow to increase the data set size of the already deployed model from 5346 to 7919 .

\section{Experimental Results}

\subsection{Sliding Window and Sampling Interval}

In order to evaluate the influence, in terms of prediction performance, of the values of $S W$ and $T_{\text {samp }}$ on the three proposed models (with reference to Figure 8), the RMSE, the MAPE, and $R^{2}$ have been calculated for each of the 210 models obtained in the training phase (i.e., 3 models for each of the 70 engineered data sets). The values of the three metrics, considering the various models, are shown, with a three-dimensional representation, in Figure 9.

It is noteworthy to remark that the values of RMSE, MAPE, and $R^{2}$ presented in Figure 9 (as well as those shown in the remainder of the paper) refer to the evaluation of the different models on the test sets (namely, set of data which have not been employed during the models' training phase). Therefore, the corresponding performance analysis refers directly to the test sets. 
Considering the results in Figure 9, the following trends, generally valid for all the evaluated NNs, can be highlighted. First, regardless of the value of $S W$, small values of $T_{\text {samp }}$ are associated with low values of RMSE (namely, a RMSE $<1^{\circ} \mathrm{C}$ for $T_{\text {samp }}=10$ or 20 or $30 \mathrm{~min}$, as shown in Figure 9a,d,g). On the other hand, high values of RMSE and, thus, degraded prediction performance, are obtained for higher values of $T_{\text {samp }}$ (e.g., $T_{\text {samp }}=120 \mathrm{~min}$ ). Second, the MAPE metric shows the same trend in all cases (as shown in Figure $9 \mathrm{~b}, \mathrm{e}, \mathrm{h})$. Third, $\mathrm{R}^{2}$ is higher the smaller $T_{\text {samp }}$ is (i.e., $T_{\text {samp }}=10 \mathrm{~min}$ ), but decreases for increasing values of $T_{\text {samp. }}$. Moreover, increasing $S W$ leads to an increase of $R^{2}$ (as shown in Figure 9c,f,i).

The first trend is also confirmed by the bi-dimensional charts shown in Figure 10, in which the prediction performance of the three NN-based models, in terms of RMSE, is shown as a function of either $S W$ (with $T_{\text {samp }}$ as a parameter) or $T_{\text {samp }}$ (with $S W$ as a parameter). In other words, the plots in Figure 10 are obtained by projecting the threedimensional plots in Figure 9 onto the two vertical planes.

Furthermore, the minimum, maximum, and average values of the three selected evaluation metrics, over the 210 considered models, are listed in Table 3, in detail with the corresponding design parameters (namely, $S W$ and $T_{\text {samp }}$ ) referring to minimum and maximum values. As can be seen from Figure 9 and Table 3, some of the considered models reach a satisfactory prediction performance on test sets while, on the other hand, some others are less accurate. An example of less accurate model is the ANN-based model trained with $\left(T_{\text {samp }}, S W\right)=(120,3)$ which, on the test set, is characterized by RMSE $=4.561{ }^{\circ} \mathrm{C}$, MAPE $=16.35 \%$, and $R^{2}=0.699$.

Less accurate models—namely, those with RMSE $>1{ }^{\circ} \mathrm{C}$, MAPE $>3 \%$, and $\mathrm{R}^{2}<0.980$ on at least one test set-will not be considered in the following analysis, although their experimental results have been included in Figure 9. In fact, since one of the goals of this paper is to discuss the influence of the dimension of the sliding window and the value of the sampling interval on the model prediction performance exhaustively, even less-performing models have been considered in Figure 9 and discussed in the previous paragraphs.

Table 3. Minimum (min), maximum (max), and average (avg) values of RMSE, MAPE, and $\mathrm{R}^{2}$ obtained over the 210 trained models.

\begin{tabular}{ccccccccccc}
\hline \multirow{2}{*}{ NN Arch. Type } & \multicolumn{3}{c}{${\text { RMSE }\left[{ }^{\circ} \mathrm{C}\right]}^{*}$} & \multicolumn{3}{c}{ MAPE [\%] } & \multicolumn{3}{c}{$\mathbf{R}^{2}$} \\
\cline { 3 - 11 } & & Value & $T_{\text {samp }}$ & SW & Value & $T_{\text {samp }}$ & $S W$ & Value & $T_{\text {samp }}$ & $S W$ \\
\hline \multirow{3}{*}{ ANN } & Min & 0.402 & 10 & 5 & 1.03 & 10 & 4 & 0.699 & 120 & 3 \\
& Max & 4.561 & 120 & 3 & 16.35 & 120 & 3 & 0.998 & 10 & 4,5 \\
& Avg & 1.52 & N/A & N/A & 4.29 & N/A & N/A & 0.96 & N/A & N/A \\
\hline \multirow{3}{*}{ RNN } & Min & 0.290 & 10 & 5 & 0.87 & 10 & 5 & 0.776 & 120 & 3 \\
& Max & 3.933 & 120 & 3 & 14.14 & 120 & 3 & 0.999 & 10 & 5 \\
& Avg & 1.45 & N/A & N/A & 4.10 & N/A & N/A & 0.96 & N/A & N/A \\
\hline \multirow{3}{*}{ LSTM } & Min & 0.294 & 10 & 5 & 0.89 & 10 & 5 & 0.766 & 120 & 3 \\
& Max & 4.024 & 120 & 3 & 14.08 & 120 & 3 & 0.999 & 10 & 5 \\
& Avg & 1.46 & N/A & N/A & 4.17 & N/A & N/A & 0.96 & N/A & N/A \\
\hline
\end{tabular}




\section{LSTM-based models}

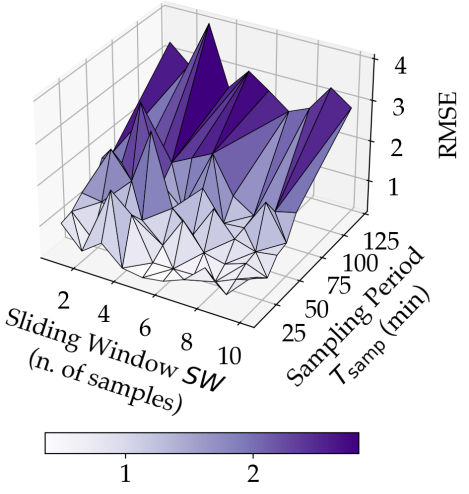

(a)

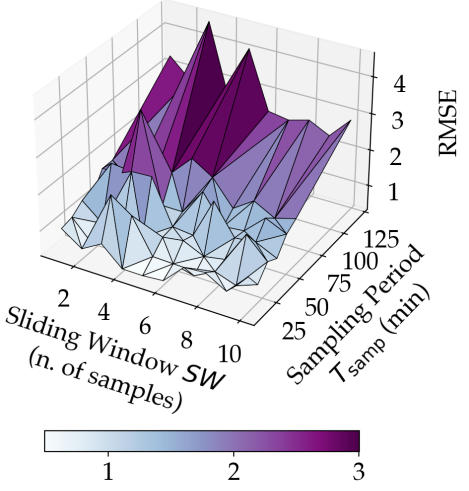

(d)

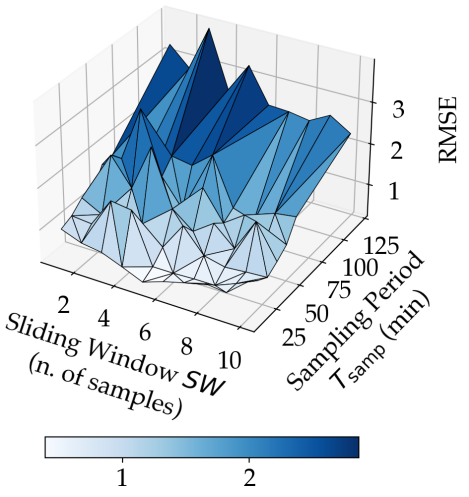

(g)

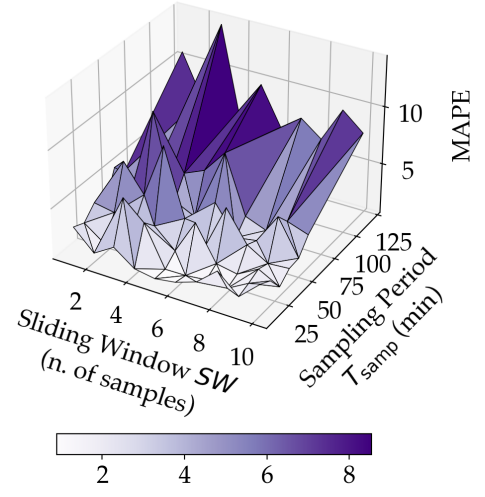

(b)

ANN-based models

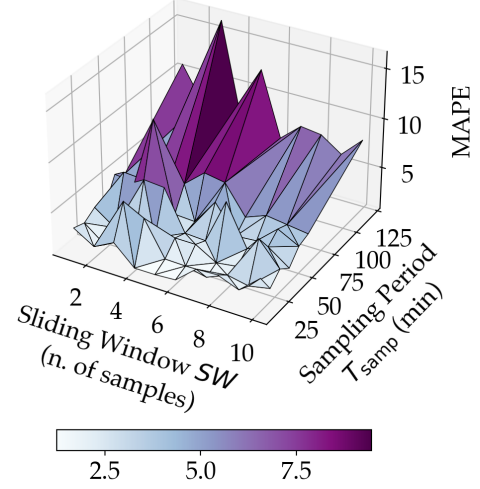

(e)

RNN-based models

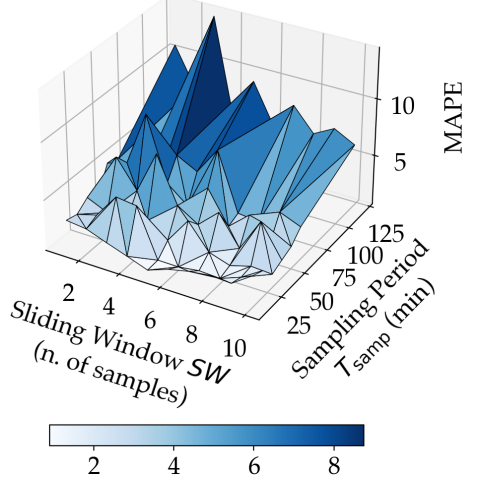

(h)

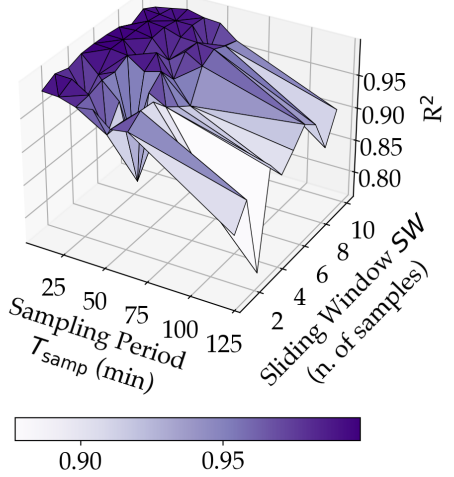

(c)

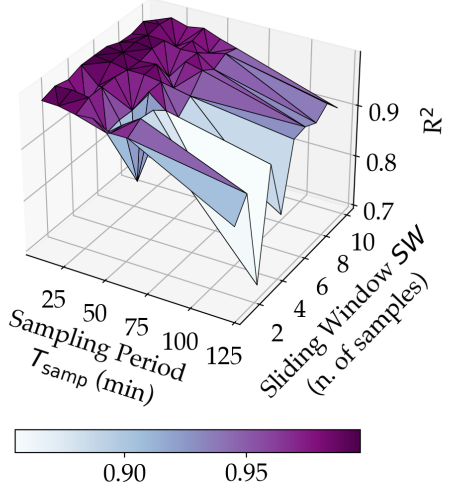

(f)

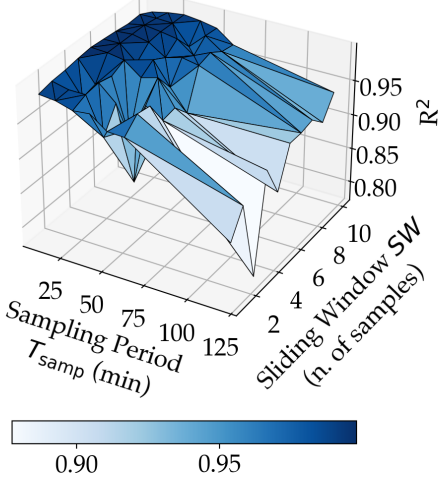

(i)

Figure 9. Experimental results with the three proposed NN-based models, namely, LSTM (a,b,c), ANN (d,e,f), and RNN $(\mathbf{g}, \mathbf{h}, \mathbf{i})$, for different values of $S W$ and $T_{\text {samp }}$, in terms of RMSE $(\mathbf{a}, \mathbf{d}, \mathbf{g}), \operatorname{MAPE}(\mathbf{b}, \mathbf{e}, \mathbf{h})$ and $\mathrm{R}^{2}(\mathbf{c}, \mathbf{f}, \mathbf{i})$. 

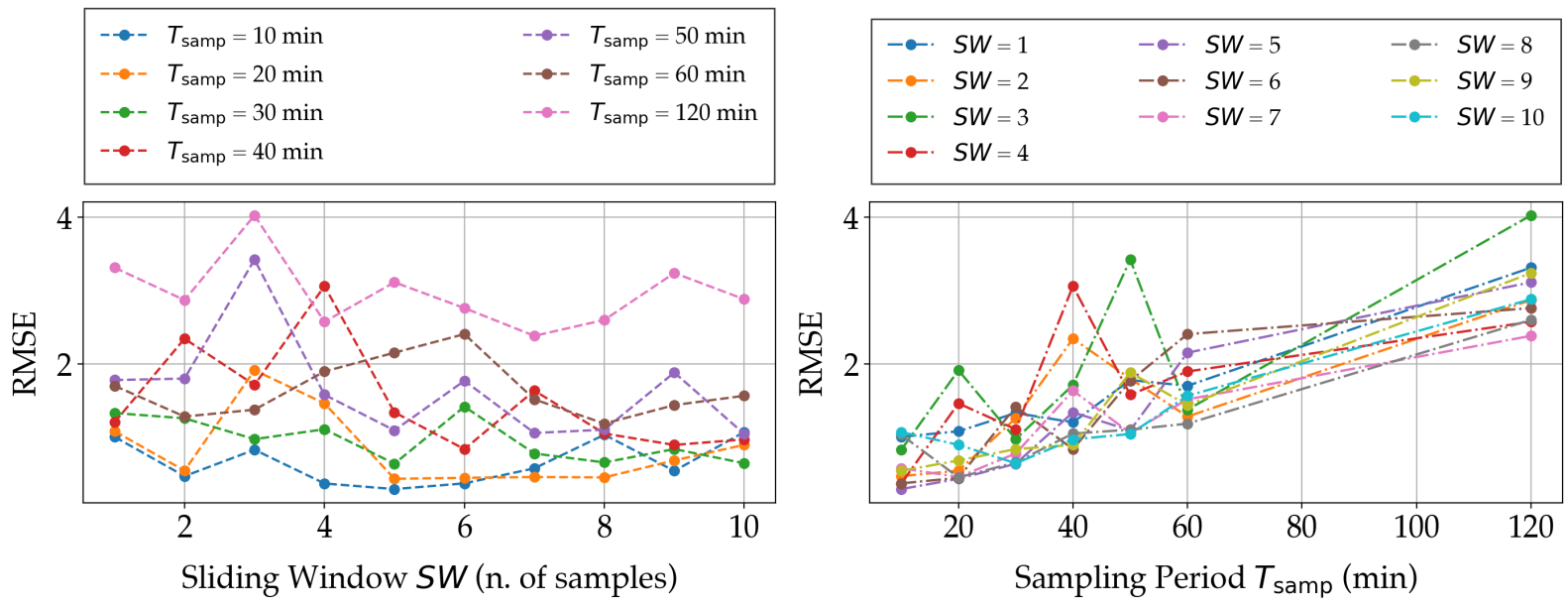

(a) LSTM-based models.
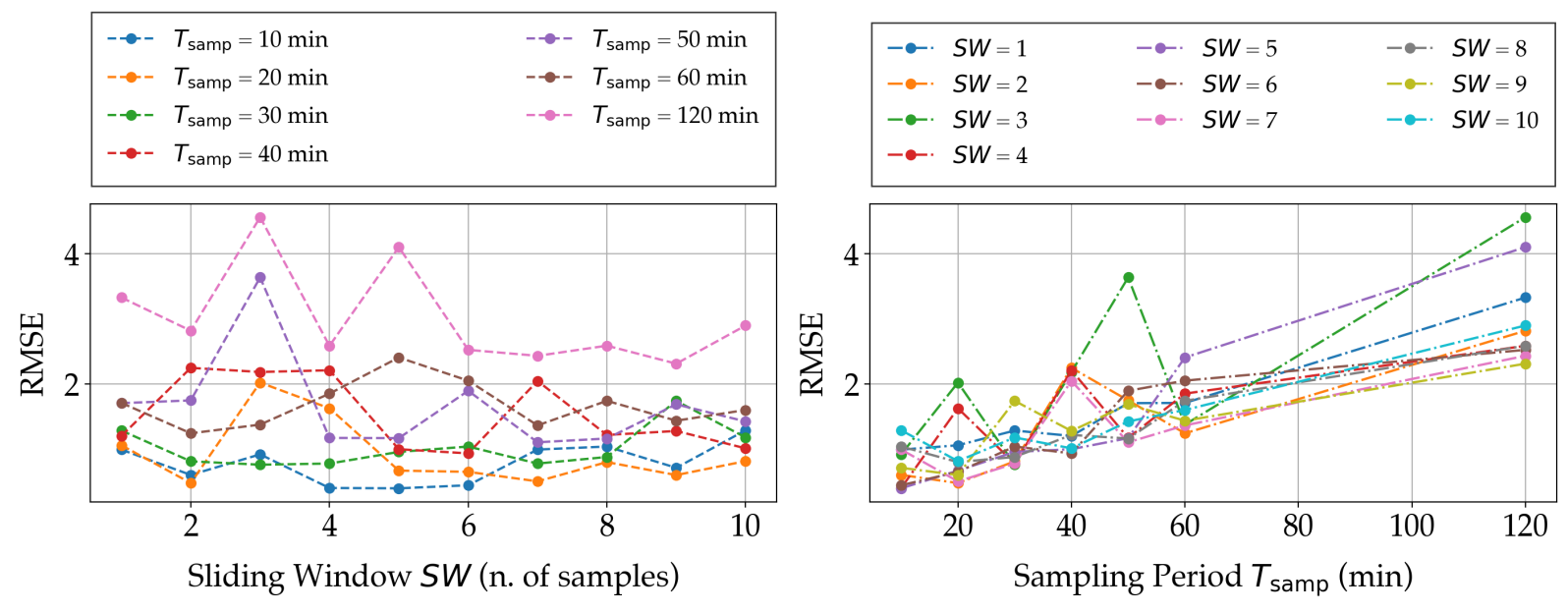

(b) ANN-based models.
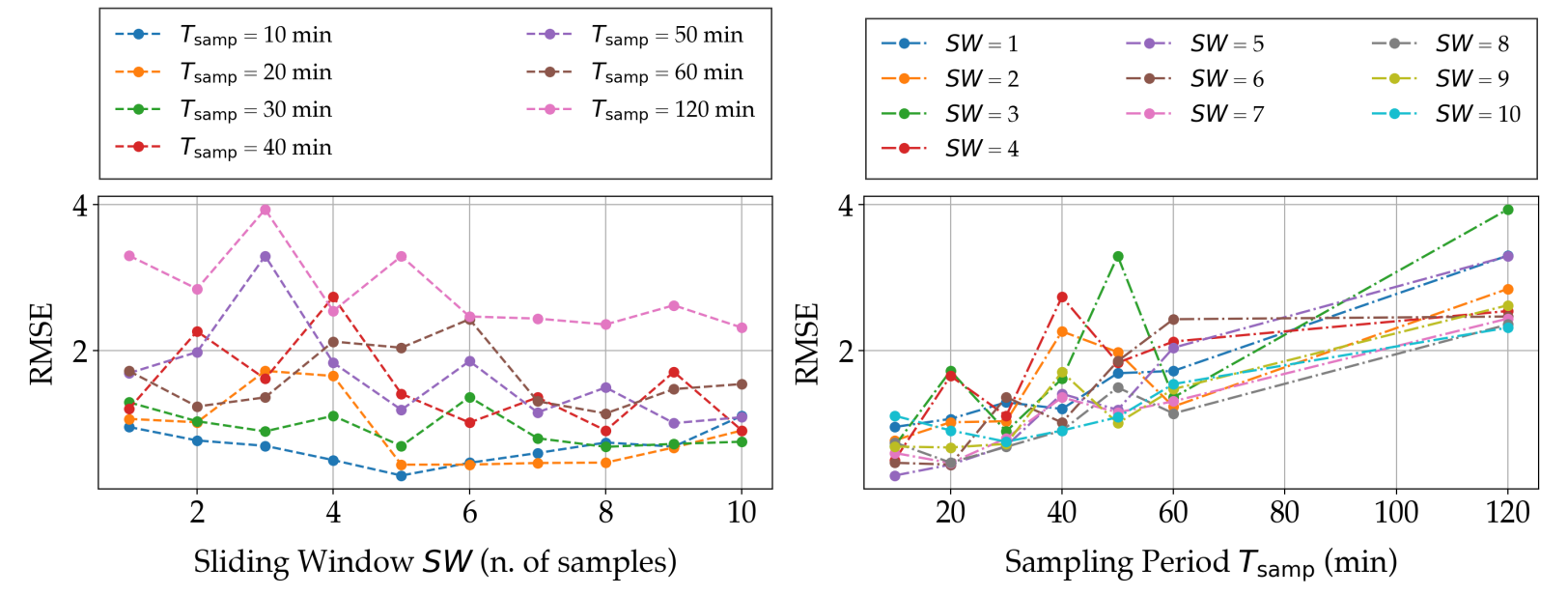

(c) RNN-based models.

Figure 10. Experimental results, in terms of RMSE, on the three proposed NN-based models, namely, (a) LSTM, (b) ANN, and (c) RNN, for different values of $S W$ and $T_{\text {samp. }}$. 


\subsection{NN Architecture}

In order to fairly compare the prediction performance of the proposed NN architectures (namely, LSTM, RNN, and ANN) over a subset of the 70 data sets generated in the previous phases, 17 models, trained on the same 17 data sets, have been selected for each type of $\mathrm{NN}$ architecture. In detail, all the data sets $\mathcal{D}_{S W}^{\left(T_{\text {samp }}\right)}$ in correspondence to which the LSTM, RNN, and ANN models trained with $T_{\text {samp }} \in\{10,20,30, \ldots, 120\}$ and $S W \in\{1,2, \ldots, 10\}$ have RMSE $\leq 1{ }^{\circ} \mathrm{C}, \mathrm{MAPE} \leq 3 \%$, and $\mathrm{R}^{2} \geq 0.980$ on test sets, have been selected. The resulting prediction performance is summarized in Table 4.

Table 4. Prediction performances of the three proposed models on a reduced selection of $S W$ and $T_{\text {samp }}$ (those which are better performing).

\begin{tabular}{lcccccccccccc}
\hline \multirow{2}{*}{$\begin{array}{c}\text { Data } \\
\text { Set }\end{array}$} & \multirow{2}{*}{$T_{\text {samp }}$} & SW & \multicolumn{3}{c}{ RMSE [ $\left.{ }^{\circ} \mathrm{C}\right]$} & \multicolumn{3}{c}{ MAPE [\%] } & \multicolumn{3}{c}{$\mathbf{R}^{2}$} \\
\cline { 3 - 11 } & & & LSTM & RNN & ANN & LSTM & RNN & ANN & LSTM & RNN & ANN \\
\hline $\mathcal{D}_{2}^{(10)}$ & 10 & 2 & 0.470 & 0.769 & 0.608 & 1.42 & 2.80 & 1.91 & 0.997 & 0.992 & 0.995 \\
$\mathcal{D}_{3}^{(10)}$ & 10 & 3 & 0.830 & 0.696 & 0.923 & 2.51 & 2.14 & 2.98 & 0.991 & 0.994 & 0.989 \\
$\mathcal{D}_{4}^{(10)}$ & 10 & 4 & 0.370 & 0.501 & 0.407 & 1.19 & 1.52 & 1.03 & 0.998 & 0.997 & 0.998 \\
$\mathcal{D}_{5}^{(10)}$ & 10 & 5 & 0.294 & 0.289 & 0.402 & 0.89 & 0.87 & 1.04 & 0.999 & 0.999 & 0.998 \\
$\mathcal{D}_{6}^{(10)}$ & 10 & 6 & 0.371 & 0.464 & 0.449 & 1.16 & 1.53 & 1.18 & 0.998 & 0.997 & 0.997 \\
$\mathcal{D}_{7}^{(10)}$ & 10 & 7 & 0.577 & 0.598 & 0.997 & 1.78 & 1.94 & 2.95 & 0.996 & 0.996 & 0.987 \\
$\mathcal{D}_{9}^{(10)}$ & 10 & 9 & 0.542 & 0.685 & 0.717 & 1.69 & 2.08 & 2.23 & 0.996 & 0.994 & 0.993 \\
\hline $\mathcal{D}_{5}^{(20)}$ & 20 & 5 & 0.434 & 0.438 & 0.674 & 0.90 & 0.93 & 1.68 & 0.998 & 0.998 & 0.994 \\
$\mathcal{D}_{6}^{(20)}$ & 20 & 6 & 0.447 & 0.439 & 0.655 & 0.93 & 0.91 & 1.63 & 0.997 & 0.998 & 0.995 \\
$\mathcal{D}_{7}^{(20)}$ & 20 & 7 & 0.458 & 0.461 & 0.509 & 1.13 & 1.16 & 1.17 & 0.997 & 0.997 & 0.997 \\
$\mathcal{D}_{8}^{(20)}$ & 20 & 8 & 0.453 & 0.466 & 0.805 & 0.98 & 1.07 & 2.06 & 0.997 & 0.997 & 0.992 \\
$\mathcal{D}_{9}^{(20)}$ & 20 & 9 & 0.684 & 0.674 & 0.606 & 2.00 & 1.77 & 1.64 & 0.994 & 0.994 & 0.995 \\
$\mathcal{D}_{10}^{(20)}$ & 20 & 10 & 0.897 & 0.907 & 0.820 & 2.80 & 2.94 & 2.08 & 0.990 & 0.990 & 0.992 \\
\hline $\mathcal{D}_{3}^{(30)}$ & 30 & 3 & 0.974 & 0.894 & 0.765 & 2.97 & 2.66 & 1.61 & 0.987 & 0.989 & 0.992 \\
$\mathcal{D}_{5}^{(30)}$ & 30 & 5 & 0.640 & 0.693 & 0.961 & 1.72 & 1.89 & 2.90 & 0.995 & 0.994 & 0.988 \\
$\mathcal{D}_{7}^{(30)}$ & 30 & 7 & 0.778 & 0.799 & 0.782 & 1.73 & 1.97 & 1.68 & 0.993 & 0.992 & 0.992 \\
$\mathcal{D}_{8}^{(30)}$ & 30 & 8 & 0.657 & 0.682 & 0.883 & 1.39 & 1.38 & 1.99 & 0.995 & 0.994 & 0.990 \\
\hline
\end{tabular}

The experimental results (expressed in terms of RMSE, MAPE, and $\mathrm{R}^{2}$ ) obtained with the three proposed NN-based models for the selected values of $S W$ and $T_{\text {samp, }}$, are shown in Figure 11.

As can be seen in Figure 11a,b, for fixed values of $S W$ and $T_{\text {samp, }}$, there is not a NN architecture which has the best performance (in terms of RMSE and MAPE) in all cases. For example, the LSTM model has the best performance in terms of RMSE with $T_{\text {samp }}=10$ and $S W=4$, while for $T_{\text {samp }}=10$ and $S W=5$ the RNN model is the most accurate. 


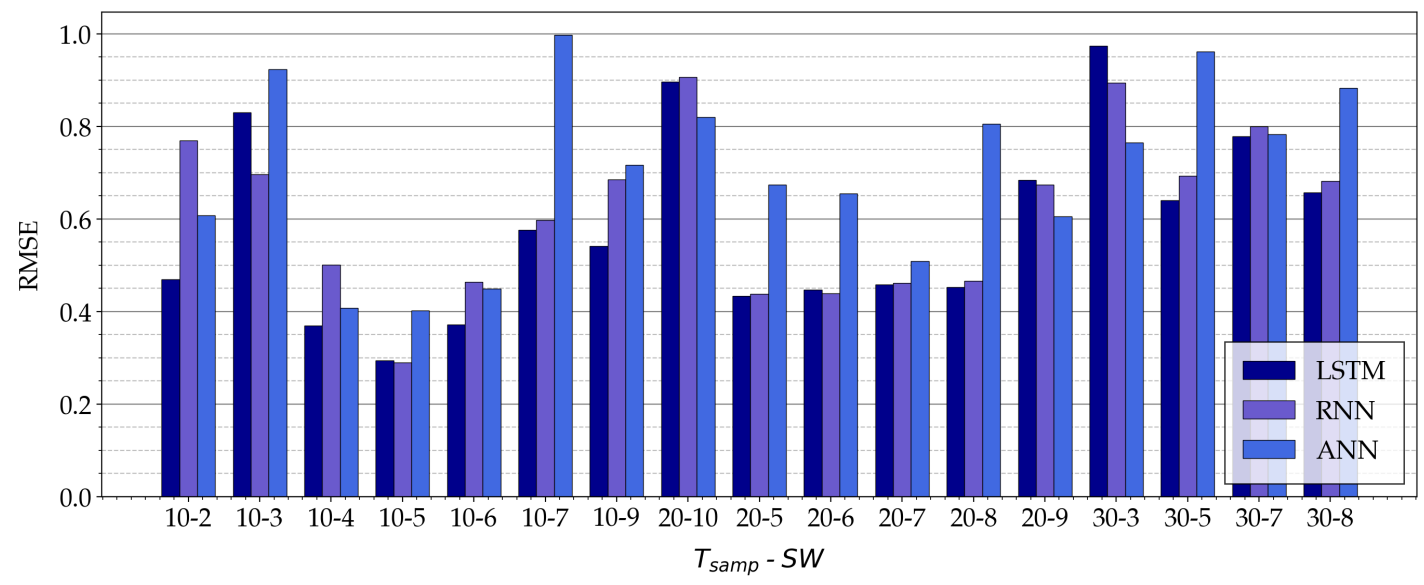

(a)

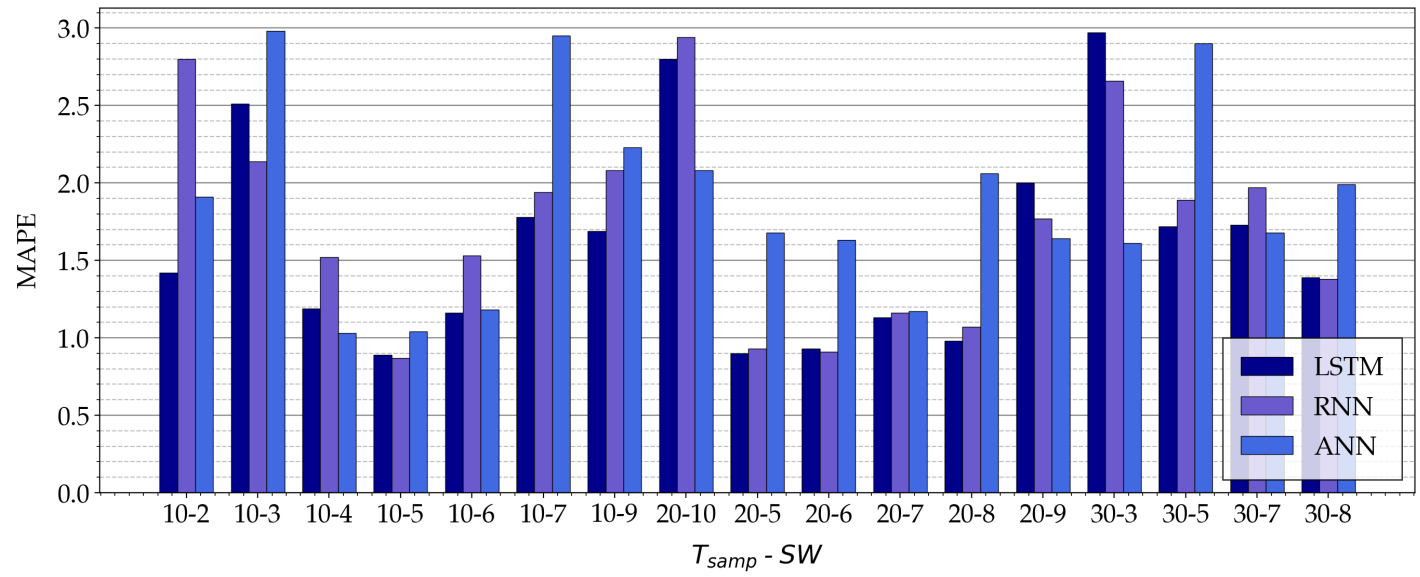

(b)

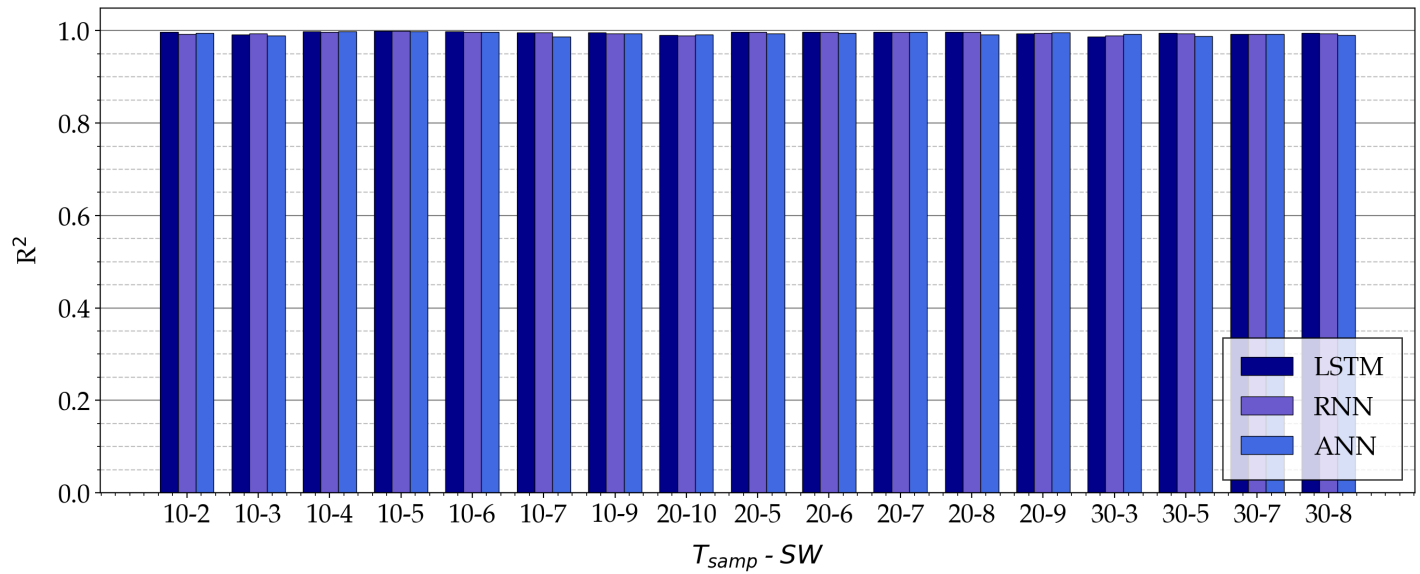

(c)

Figure 11. Experimental results on the three proposed NN-based models, namely, LSTM, ANN, and RNN, for a few relevant combination of $S W$ and $T_{\text {samp, }}$ expressed in terms of (a) RMSE, (b) MAPE, and (c) $R^{2}$.

In general, better results (in terms of the adopted metrics) are given by the following pairs of values of $\left(T_{\text {samp }}, S W\right)$ : $(10,4),(10,5),(10,6),(20,5),(20,6),(20,7)$, and $(20,8)$. For these values, RNN and LSTM models have RMSE $\leq 0.5^{\circ} \mathrm{C}$, MAPE $<1.6 \%$, and $\mathrm{R}^{2} \geq 0.997$. 
To conclude, the overall model with the best performance (with regard to the considered metrics) is the RNN model with $\left(T_{\text {samp }}, S W\right)=(10,5)$, which guarantees a RMSE $=0.289^{\circ} \mathrm{C}$, a MAPE $=0.87 \%$, and a $R^{2}=0.999$. It should be remarked that very similar results have been achieved with the LSTM model with the same values of $T_{\text {samp }}$ and $S W$ (namely, RMSE $=0.294{ }^{\circ} \mathrm{C}, \mathrm{MAPE}=0.98 \%$, and $\mathrm{R}^{2}=0.999$ ).

\subsection{Performance Analysis and Literature Comparison}

The experimental results of the model presented in [19] (obtained with the data set available in [19]), together with the results of the same model re-trained with a data set containing additional samples (as described in Section 4.5) and with the three models (ANN, RNN, and LSTM) built with the data set $\mathcal{D}_{5}^{(10)}$, are shown in Table 5. As can be seen from Table 5 that the prediction performance of the re-trained model degrades with respect to that in [19]. One possible reason behind this behavior could be that, although re-training involves additional samples (and, therefore, should be, in principle, more accurate), it may happen that the supplementary data are more unbalanced (e.g., in terms of number of collected samples per month, as shown in Figure 4), thus reducing the final accuracy. Moreover, if the model presented in [19] are compared, in terms of prediction performance, with the three models with lowest RMSE obtained with the data set $\mathcal{D}_{5}^{(10)}$ (namely: $\operatorname{LSTM}_{5}^{(10)}, \mathrm{RNN}_{5}^{(10)}$, and $\mathrm{ANN}_{5}^{(10)}$ ), one can conclude that the RMSEs of the latter are slightly lower. Indeed, the RMSE of the model in [19] is higher than the last three collected in Table 5 by at least $1{ }^{\circ} \mathrm{C}$, the MAPE is higher by approximately $3 \%$, and the $\mathrm{R}^{2}$ is lower by approximately 0.24 .

Table 5. Prediction performance and complexity of a subset of evaluated models, in terms of RMSE, MAPE, $\mathrm{R}^{2}$, accuracy (namely, number of samples predicted with RMSE lower than $1^{\circ} \mathrm{C}$ ), MAC operations, number of parameters, and NetScore of the models.

\begin{tabular}{|c|c|c|c|c|c|c|c|}
\hline Model & $\operatorname{RMSE}\left[{ }^{\circ} \mathrm{C}\right]$ & MAPE [\%] & $\mathbf{R}^{2}$ & Accuracy [\%] & $\begin{array}{c}\text { MAC } \\
\text { Operations }\end{array}$ & $\begin{array}{c}\text { Parameters } \\
\text { Number }\end{array}$ & NetScore \\
\hline Model in [19] & 1.50 & 4.91 & 0.965 & 48.87 & 1018 & 1018 & 17.05 \\
\hline Re-trained [19] & 2.28 & 6.54 & 0.931 & 34.91 & 1018 & 1018 & 3.60 \\
\hline $\operatorname{LSTM}_{5}^{(10)}$ & 0.294 & 0.89 & 0.999 & 99.18 & 22,192 & 4625 & -0.59 \\
\hline $\mathrm{RNN}_{5}^{(10)}$ & 0.289 & 0.87 & 0.999 & 99.28 & 5712 & 1361 & 25.25 \\
\hline $\mathrm{ANN}_{5}^{(10)}$ & 0.402 & 1.04 & 0.998 & 97.28 & 464 & 464 & 60.31 \\
\hline
\end{tabular}

In Table 5, the architectural and computational complexities of the considered models are evaluated. More precisely, the architectural and computational complexities can be expressed, respectively, in terms of number of parameters to be used by the model and MAC operations (see Section 2). Analyzing the results in Table 5, the LSTM-based model (namely, $\operatorname{LSTM}_{5}^{(10)}$ ) is the most architecturally and computationally complex (with respect to the other NNs). The RNN-based model (namely, $\mathrm{RNN}_{5}^{(10)}$ ) ranks second and is then followed by the model presented in [19] and its re-trained version. The $\mathrm{ANN}_{5}^{(10)}$ model is the "lightest" model, with only 464 parameters and 464 MAC operations. In other words, $\mathrm{ANN}_{5}^{(10)}$ is the one with lowest architectural and computational complexities.

Finally, the NetScore metric (introduced in Section 2) has been calculated for the 5 models detailed in Table 5, with the goal to identify which model reaches the best tradeoff between prediction performance and complexity. With reference to Equation (5), one of the parameters required to calculate the NetScore (for a target NN model $\mathcal{N}$ ) is the model's accuracy $a(\mathcal{N})$. This accuracy is an evaluation metric generally adopted in classification tasks, which can be defined, over a target data set, as the percentage of samples correctly attributed to their classes by a predictor. Since the air temperature forecasting is practically a regression task and, for this reason, the canonical definition of the accuracy cannot be 
applied for the evaluation of the models, the authors re-define the concept of accuracyand, thus, the meaning of $a(\mathcal{N})$ in Equation (5)—in order to calculate the NetScore metric for the models. In detail, the authors define a threshold parameter $\mathcal{T}$ and an indicator function $\mathcal{I}$ which may assume, for each sample $\underline{d}(k)=(\underline{x}(k), y(k))$ in the test subset of the model to be evaluated, a binary value as follows:

$$
\mathcal{I}(\underline{d}(k))=\mathcal{I}(y(k), \hat{y}(k))=\mathcal{U}(\mathcal{T}-|y(k)-\hat{y}(k)|)= \begin{cases}1 & \text { if }|y(k)-\hat{y}(k)|<\mathcal{T} \\ 0 & \text { otherwise }\end{cases}
$$

where: $\mathcal{U}(\cdot)$ is the unit step function; $|\cdot|$ is the modulo operator; $\underline{d}(k)$ is the $k$-th entry in the test subset; $\underline{x}(k)$ is the vector of the input samples of the test subset entry $\underline{d}(k) ; y(k)$ and $\hat{y}(k)$ are the actual (in the entry $\underline{d}(k)$ ) and the forecast by the $\mathrm{NN}$ (with input $\underline{x}(k)$ ) values of air temperature for the $k$-th test subset entry, respectively; and $\mathcal{T}$ is a threshold value.

Therefore, the accuracy $a(\mathcal{N})$ for the model $\mathcal{N}$ over all the samples in the test subset is defined as follows:

$$
a(\mathcal{N})=\frac{100}{N_{\mathrm{tst}}} \sum_{r=1}^{N_{\mathrm{tst}}} \mathcal{I}(y(k), \hat{y}(k))
$$

where $N_{\text {tst }}$ is the number of samples in the test subset; $y(k)$ and $\hat{y}(k)$ are the actual and the forecast values (of air temperature) for the $k$-th test subset entry, respectively.

The value of $\mathcal{T}$ is set to $1{ }^{\circ} \mathrm{C}$ : from a conceptual point of view, this means that a future air temperature value $\hat{y}(k)$ forecast by the model is considered as correct (e.g., $\mathcal{I}(y(k), \hat{y}(k))=1$ ) if the absolute value of the difference between actual and forecast air temperature values $(|y(k)-\hat{y}(k)|)$ is lower than $1{ }^{\circ} \mathrm{C}$. The exponential coefficients of the NetScore metric are set to their default values, namely $\alpha=2$ and $\beta=\gamma=0.5$. The corresponding NetScore values are listed in Table 5.

As can be seen from the results in Table 5, the highest NetScore is reached by the $\mathrm{ANN}_{5}^{(10)}$ model, followed by the $\mathrm{RNN}_{5}^{(10)}$ model, and then by the model the authors presented in [19]. On the other hand, the re-trained version of [19] and the LSTM$_{5}^{(10)}$ model return the lowest NetScore, thus highlighting that the trade-off between prediction performance and complexity achieved by these models is rather unbalanced. Indeed, the prediction performance of the re-trained version of [19] is lower than that of the $\mathrm{LSTM}_{5}^{(10)}$ model (e.g., with RMSE equal to $2.28^{\circ} \mathrm{C}$ and $0.294^{\circ} \mathrm{C}$, respectively). On the other hand, computational and architectural complexities of the re-trained version of [19] are significantly lower than those of the $\mathrm{LSTM}_{5}^{(10)}$ model, with a ratio between the number of MAC operations of the re-trained model from [19] and $\operatorname{LSTM}_{5}^{(10)}$ approximately equal to 1:22. Overall, the $\mathrm{ANN}_{5}^{(10)}$ is the model to be preferred for execution on edge devices.

With regard to the reference works listed in Table 1, it can be noted that the prediction performance of the three best models proposed in this paper (namely, $\mathrm{LSTM}_{5}^{(10)}, \mathrm{RNN}_{5}^{(10)}$, and $\mathrm{RNN}_{5}^{(10)}$ ) are comparable with those provided in these works. In particular:

- the proposed NN-based models have a RMSE in the range $0.289 \div 0.402{ }^{\circ} \mathrm{C}$, lower than that in $[3,14,27,28]$ and slightly higher than that in $[15,16]$;

- the considered NN-based models have a MAPE in the range $0.87 \div 1.04 \%$, thus lower than that in [15,27];

- the value of $\mathrm{R}^{2}$ of the considered NN-based models is higher than those of all the references listed in Table 1.

Finally, as detailed in Section 2, the literature works listed in Table 1 do not provide details on the complexity of their deployed models: this prevents a direct comparison, from a complexity-performance trade-off perspective, between them and the NN-based models proposed in this paper. 


\subsection{Possible Application Scenario and Reference Architecture}

From an implementation point of view, the NN model selected in Section 5.3 (namely, $\mathrm{ANN}_{5}^{(10)}$ ) can be deployed on a real IoT edge device, denoted as Smart GW, such as a Raspberry Pi (RPi) [33], which represents a popular Single Board Computer (SBC) in EdgeAI applications [34,35]. Targeting a Smart Farming scenario, the Smart GW can be placed inside a greenhouse, which hosts also a WSN deployed to monitor the internal air temperature, and acts as a data collector for the WSN's SNs, as shown in Figure 12. More precisely, SNs in the greenhouse forward-sensed air temperature data to the Smart GW thanks to a wireless connection existing (or introduced specifically for this purpose) in the greenhouse, e.g., a Wi-Fi network. Then, the information arriving from the SNs is temporarily stored by the GW in its internal memory and, if necessary, also forwarded to the Cloud (if an Internet access point is available around the greenhouse).

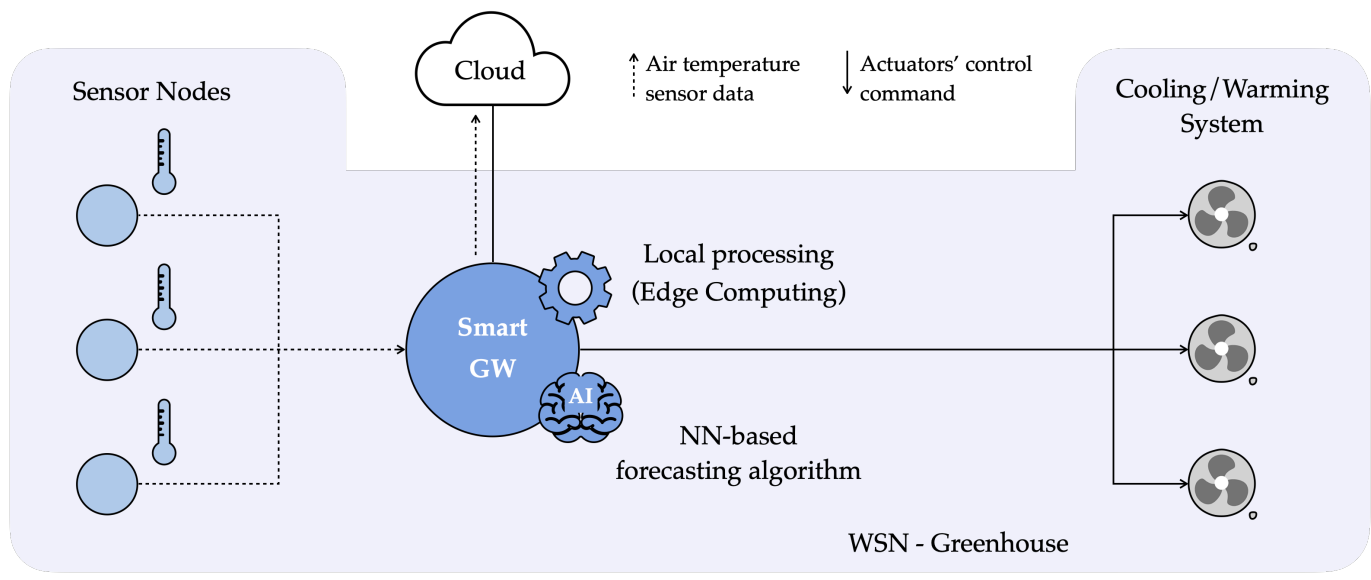

Figure 12. Possible reference architecture and application scenario for the developed forecasting model.

In a demonstration greenhouse in the AFarCloud project [30], the Smart GW is also connected with the cooling/warming system installed inside the greenhouse and can control it to regulate the internal air temperature and humidity levels. If needed, as well as when the proper number of consecutive air temperature values have been collected from $\mathrm{SNs}-\mathrm{in}$ turn, to be used as inputs for the forecasting model (e.g., 5 values for $\mathrm{ANN}_{5}^{(10)}$ ) the Smart GW runs the forecasting algorithm, obtaining a future predicted air temperature value. According to the forecast value, the Smart GW decides if the cooling/warming system needs to be activated, deactivated, or maintained in its current operational state. As an example, if the predicted air temperature value is higher than a certain threshold which cannot be exceeded inside the greenhouse, the GW can send a command to the internal cooling system requiring an opening actuation, thus avoiding the internal air temperature from reaching the unwanted (and dangerous) forecast value.

To conclude, the adoption of EdgeAI solutions has an extremely positive impact on greenhouse management, which, as previously mentioned, needs to tightly control the internal microclimate. Indeed, being able to predict the trend of internal environmental variables (e.g., air temperature), such an IoT/EdgeAI system allows to preemptively schedule actions and, thus, to more effectively pilot greenhouse's actuators to maintain (within the greenhouse) the best-growing conditions for the internal cultivation. Moreover, executing AI algorithms and taking decisions at the edge, rather than in the Cloud, makes greenhouse management more robust (against connectivity problems) and reduces latency. As a last comment, the proposed IoT-oriented EdgeAI NN-based forecasting can be effectively integrated into the LoRaFarM platform [8] simply as a new additional software module. 


\section{Conclusions}

In this paper, the authors have presented an NN-based approach, based on time series, expedient to design a forecasting algorithm to be embedded into a Smart GW, in turn acting as a data collector for $\mathrm{SNs}$ measuring air temperature inside a greenhouse. In comparison to the approach in [19], based on an ANN-based prediction model to predict temperature values considering weather conditions external to the greenhouse as input sources, in this paper the authors have considered indoor air temperature values, collected inside the greenhouse, as NN models' input variables. In detail, three types of NN architecturesnamely, LSTM, RNN, and ANN—have been investigated to determine the one with the best performance and the lowest complexity (from both computational and architectural viewpoints). This is fundamental to run a NN model on an edge device with constrained capabilities-namely, the Smart GW presented and discussed in [19].

According to the obtained experimental results, it can be concluded that the three proposed models (namely, LSTM, RNN, and ANN) reach a prediction performance comparable to that of literature works improve over that of the model proposed in [19]. In particular, the three best performing algorithms, obtained with a sampling interval $T_{\text {samp }}=10 \mathrm{~min}$ and using $S W=5$ input variables, reach a RMSE in the range of $0.289 \div 0.402{ }^{\circ} \mathrm{C}$, a MAPE in the range of $0.87 \div 1.04 \%$, and a $\mathrm{R}^{2} \geq 0.997$. Moreover, the results show that the ANN-based model has lower computational complexity, in terms of the number of MAC operations and parameters to store, than the one proposed in [19]. Overall, $\mathrm{ANN}_{5}^{(10)}$ is the $\mathrm{NN}$ model guaranteeing the best performance-complexity trade-off. In general, the design and implementation of NN-based accurate prediction algorithms, yet with a computational complexity compatible with the processing resources of IoT edge devices, is an interesting and promising research topic, not extensively discussed in recent literature. This is even more true for the greenhouse's internal variables forecasting, which this paper has tried to address in the more comprehensive possible way.

As a final remark, future research directions may involve the performance evaluation of the proposed NN-based algorithms on different types of IoT edge devices, based on relevant metrics for the algorithms' online execution. To this end, illustrative interesting performance metrics are the following: (i) the time required by the device to run the algorithm in a real scenario; (ii) the (flash) memory space the device needs to store the model, as well as the RAM memory required to run it; and (iii) the IoT edge device's power consumption when running the NN-based algorithms to forecast a future air temperature value. Another possible direction includes the application of the same methodology followed in this paper (in order to build an air temperature forecasting model) to develop algorithms able to predict other types of inner variables (rather than air temperature) which, in turn, are relevant for greenhouse cultivation and, thus, have to be monitored and controlled (e.g., air humidity).

Author Contributions: conceptualization, G.C., L.D. and G.F.; methodology, G.C., L.D. and G.F.; software, G.C., L.D. and G.F.; validation, G.C., L.D. and G.F.; formal analysis, G.C., L.D. and G.F.; investigation, G.C., L.D. and G.F.; resources, G.C., L.D. and G.F.; data curation, G.C., L.D. and G.F.; writing — original draft preparation, G.C., L.D. and G.F.; writing—review and editing, G.C., L.D. and G.F.; visualization, G.C., L.D. and G.F.; supervision, G.C., L.D. and G.F.; project administration, G.C., L.D. and G.F.; funding acquisition, G.F. All authors have read and agreed to the published version of the manuscript.

Funding: This work has received funding from the European Union's Horizon 2020 research and innovation program ECSEL Joint Undertaking (JU) under Grant Agreement No. 783221, AFarCloud project-“Aggregate Farming in the Cloud" and Grant Agreement No. 876038, InSecTT project"Intelligent Secure Trustable Things." The work of L.D. is partially funded by the University of Parma, under "Iniziative di Sostegno alla Ricerca di Ateneo" program, "Multi-interface IoT sYstems for Multi-layer Information Processing" (MIoTYMIP) project. The work of G.C. is funded by the Regione Emilia Romagna, under "Sistemi IoT per la raccolta e l'elaborazione dei dati efficienti in agricoltura di precisione e sostenibile (AgrIoT)" Ph.D. scholarship. The JU received support from the European Union's Horizon 2020 research and innovation programme and the nations involved in 
the mentioned projects. The work reflects only the authors' views; the European Commission is not responsible for any use that may be made of the information it contains.

Institutional Review Board Statement: Not applicable.

Informed Consent Statement: Not applicable.

Data Availability Statement: Not applicable.

Conflicts of Interest: The authors declare no conflict of interest. The funders had no role in the design of the study; in the collection, analyses, or interpretation of data; in the writing of the manuscript, or in the decision to publish the results.

\section{Abbreviations}

The following abbreviations are used in this manuscript:

\begin{tabular}{|c|c|}
\hline AFarCloud & Aggregate Farming in the Cloud \\
\hline AI & Artificial Intelligence \\
\hline ANN & Artificial Neural Network \\
\hline $\mathrm{BP}$ & Back Propagation \\
\hline CGA & Conjugate Gradient Algorithm \\
\hline DL & Deep Learning \\
\hline DNN & Deep Neural Network \\
\hline FaaS & Farm-as-a-Service \\
\hline GW & Gateway \\
\hline ICT & Information and Communication Technology \\
\hline IoT & Internet of Things \\
\hline LM & Levenberg-Marquardt \\
\hline LSTM & Long Short-Term Memory \\
\hline MAC & Multiply-ACcumulate \\
\hline MAPE & Mean Absolute Percentage Error \\
\hline ML & Machine Learning \\
\hline MLP & Multi-Layer Perceptron \\
\hline NARX & Nonlinear AutoRegressive with eXternal input \\
\hline NN & Neural Network \\
\hline PSO & Particle Swarm Optimization \\
\hline $\mathrm{R}^{2}$ & Coefficient of determination \\
\hline RBF & Radial Basis Function \\
\hline ReLU & Rectified Linear Unit \\
\hline RMSE & Root Mean Squared Error \\
\hline RNN & Recurrent Neural Network \\
\hline SA & Smart Agriculture \\
\hline SBC & Single Board Computer \\
\hline SF & Smart Farming \\
\hline SN & Sensor Node \\
\hline UI & User Interface \\
\hline WSN & Wireless Sensor Network \\
\hline
\end{tabular}




\section{References}

1. Codeluppi, G.; Cilfone, A.; Davoli, L.; Ferrari, G. VegIoT Garden: A modular IoT Management Platform for Urban Vegetable Gardens. In Proceedings of the IEEE International Workshop on Metrology for Agriculture and Forestry (MetroAgriFor), Portici, Italy, 24-26 October 2019; pp. 121-126. [CrossRef]

2. Kumar, A.; Tiwari, G.N.; Kumar, S.; Pandey, M. Role of Greenhouse Technology in Agricultural Engineering. Int. J. Agric. Res. 2010, 5, 779-787. [CrossRef]

3. Francik, S.; Kurpaska, S. The Use of Artificial Neural Networks for Forecasting of Air Temperature inside a Heated Foil Tunnel. Sensors 2020, 20, 652. [CrossRef] [PubMed]

4. Escamilla-García, A.; Soto-Zarazúa, G.M.; Toledano-Ayala, M.; Rivas-Araiza, E.; Gastélum-Barrios, A. Applications of Artificial Neural Networks in Greenhouse Technology and Overview for Smart Agriculture Development. Appl. Sci. 2020, $10,3835$. [CrossRef]

5. Bot, G. Physical Modeling of Greenhouse Climate. IFAC Proc. Vol. 1991, 24, 7-12. [CrossRef]

6. Belli, L.; Cirani, S.; Davoli, L.; Melegari, L.; Mónton, M.; Picone, M. An Open-Source Cloud Architecture for Big Stream IoT Applications. In Interoperability and Open-Source Solutions for the Internet of Things: International Workshop, FP7 OpenIoT Project, Held in Conjunction with SoftCOM 2014, Split, Croatia, 18 September 2014, Invited Papers; Podnar Žarko, I., Pripužić, K., Serrano, M., Eds.; Springer International Publishing: Berlin/Heidelberg, Germany, 2015; pp. 73-88. [CrossRef]

7. Kochhar, A.; Kumar, N. Wireless sensor networks for greenhouses: An end-to-end review. Comput. Electron. Agric. 2019, 163, 104877. [CrossRef]

8. Codeluppi, G.; Cilfone, A.; Davoli, L.; Ferrari, G. LoRaFarM: A LoRaWAN-Based Smart Farming Modular IoT Architecture. Sensors 2020, 20, 2028. [CrossRef] [PubMed]

9. Abbasi, M.; Yaghmaee, M.H.; Rahnama, F. Internet of Things in agriculture: A survey. In Proceedings of the 3rd International Conference on Internet of Things and Applications (IoT), Isfahan, Iran, 17-18 April 2019; pp. 1-12. [CrossRef]

10. Davoli, L.; Belli, L.; Cilfone, A.; Ferrari, G. Integration of Wi-Fi mobile nodes in a Web of Things Testbed. ICT Express 2016, 2, 95-99. [CrossRef]

11. Tafa, Z.; Ramadani, F.; Cakolli, B. The Design of a ZigBee-Based Greenhouse Monitoring System. In Proceedings of the 7th Mediterranean Conference on Embedded Computing (MECO), Budva, Montenegro, 10-14 June 2018; pp. 1-4. [CrossRef]

12. Wiboonjaroen, M.T.W.; Sooknuan, T. The Implementation of PI Controller for Evaporative Cooling System in Controlled Environment Greenhouse. In Proceedings of the 17th International Conference on Control, Automation and Systems (ICCAS), Jeju, Korea, 18-21 October 2017; pp. 852-855. [CrossRef]

13. Zou, Z.; Bie, Y.; Zhou, M. Design of an Intelligent Control System for Greenhouse. In Proceedings of the 2nd IEEE Advanced Information Management, Communicates, Electronic and Automation Control Conference (IMCEC), Xi'an, China, 25-27 May 2018; pp. 1-1635. [CrossRef]

14. Moon, T.; Hong, S.; Young Choi, H.; Ho Jung, D.; Hong Chang, S.; Eek Son, J. Interpolation of Greenhouse Environment Data using Multilayer Perceptron. Comput. Electron. Agric. 2019, 166, 105023. [CrossRef]

15. Taki, M.; Abdanan Mehdizadeh, S.; Rohani, A.; Rahnama, M.; Rahmati-Joneidabad, M. Applied machine learning in greenhouse simulation; New application and analysis. Inf. Process. Agric. 2018, 5, 253-268. [CrossRef]

16. Yue, Y.; Quan, J.; Zhao, H.; Wang, H. The Prediction of Greenhouse Temperature and Humidity Based on LM-RBF Network. In Proceedings of the IEEE International Conference on Mechatronics and Automation (ICMA), Changchun, China, 5-8 August 2018; pp. 1537-1541. [CrossRef]

17. Lee, Y.; Tsung, P.; Wu, M. Techology Trend of Edge AI. In Proceedings of the International Symposium on VLSI Design, Automation and Test (VLSI-DAT), Hsinchu, Taiwan, 16-19 April 2018; pp. 1-2. [CrossRef]

18. Wong, A. NetScore: Towards Universal Metrics for Large-Scale Performance Analysis of Deep Neural Networks for Practical On-Device Edge Usage. In Image Analysis and Recognition; Karray, F., Campilho, A., Yu, A., Eds.; Springer International Publishing: Cham, Switzerland, 2019; pp. 15-26. [CrossRef]

19. Codeluppi, G.; Cilfone, A.; Davoli, L.; Ferrari, G. AI at the Edge: A Smart Gateway for Greenhouse Air Temperature Forecasting. In Proceedings of the IEEE International Workshop on Metrology for Agriculture and Forestry (MetroAgriFor), Trento, Italy, 4-6 November 2020; pp. 348-353. [CrossRef]

20. Abiodun, O.I.; Jantan, A.; Omolara, A.E.; Dada, K.V.; Mohamed, N.A.; Arshad, H. State-of-the-art in artificial neural network applications: A survey. Heliyon 2018, 4, e00938. [CrossRef] [PubMed]

21. Cifuentes, J.; Marulanda, G.; Bello, A.; Reneses, J. Air Temperature Forecasting Using Machine Learning Techniques: A Review. Energies 2020, 13, 4215. [CrossRef]

22. Kavlakoglu, E. AI vs. Machine Learning vs. Deep Learning vs. Neural Networks: What's the Difference? Available online: https:/ / www.ibm.com/cloud/blog/ai-vs-machine-learning-vs-deep-learning-vs-neural-networks (accessed on 2 March 2021).

23. Ferrero Bermejo, J.; Gómez Fernández, J.F.; Olivencia Polo, F.; Crespo Márquez, A. A Review of the Use of Artificial Neural Network Models for Energy and Reliability Prediction. A Study of the Solar PV, Hydraulic and Wind Energy Sources. Appl. Sci 2019, 8, 1844. [CrossRef]

24. Gardner, M.; Dorling, S. Artificial neural networks (the multilayer perceptron)-A review of applications in the atmospheric sciences. Atmos. Environ. 1998, 32, 2627-2636. [CrossRef] 
25. Bengio, Y.; Simard, P.; Frasconi, P. Learning long-term dependencies with gradient descent is difficult. IEEE Trans. Neural Netw. 1994, 5, 157-166. [CrossRef] [PubMed]

26. Hochreiter, S.; Schmidhuber, J. Long Short-Term Memory. Neural Comput. 1997, 9, 1735-1780. [CrossRef] [PubMed]

27. Hongkang, W.; Li, L.; Yong, W.; Fanjia, M.; Haihua, W.; Sigrimis, N. Recurrent Neural Network Model for Prediction of Microclimate in Solar Greenhouse. IFAC-PapersOnLine 2018, 51, 790-795. [CrossRef]

28. Jung, D.H.; Seok Kim, H.; Jhin, C.; Kim, H.J.; Hyun Park, S. Time-serial analysis of deep neural network models for prediction of climatic conditions inside a greenhouse. Comput. Electron. Agric. 2020, 173, 105402. [CrossRef]

29. Taki, M.; Ajabshirchi, Y.; Ranjbar, S.F.; Rohani, A.; Matloobi, M. Heat transfer and MLP neural network models to predict inside environment variables and energy lost in a semi-solar greenhouse. Energy Build. 2016, 110, 314-329. [CrossRef]

30. Aggregate Farming in the Cloud (AFarCloud) H2020 Project. Available online: http://www.afarcloud.eu (accessed on 14 February 2021).

31. Podere Campáz-Produzioni Biologiche. Available online: https://www.poderecampaz.com (accessed on 1 February 2020).

32. Chollet, F. Keras. 2015 Available online: https:/ / keras.io (accessed on 15 May 2021).

33. Raspberry Pi. Available online: https://www.raspberrypi.org/ (accessed on 1 June 2021).

34. Mazzia, V.; Khaliq, A.; Salvetti, F.; Chiaberge, M. Real-Time Apple Detection System Using Embedded Systems With Hardware Accelerators: An Edge AI Application. IEEE Access 2020, 8, 9102-9114. [CrossRef]

35. Shadrin, D.; Menshchikov, A.; Ermilov, D.; Somov, A. Designing Future Precision Agriculture: Detection of Seeds Germination Using Artificial Intelligence on a Low-Power Embedded System. IEEE Sens. J. 2019, 19, 11573-11582. [CrossRef] 